\title{
Temporal variations of the outer atmosphere and the dust shell of the carbon-rich Mira variable V Ophiuchi probed with VLTI/MIDI ${ }^{\star \star \star \star}$
}

\author{
K. Ohnaka ${ }^{1}$, T. Driebe ${ }^{1}$, G. Weigelt ${ }^{1}$, and M. Wittkowski ${ }^{2}$ \\ ${ }^{1}$ Max-Planck-Institut für Radioastronomie, Auf dem Hügel 69, 53121 Bonn, Germany \\ e-mail: kohnaka@mpifr-bonn.mpg.de \\ ${ }^{2}$ European Southern Observatory, Karl-Schwarzschild-Str. 2, 85748 Garching, Germany
}

Received 23 November 2006 / Accepted 30 January 2007

\section{ABSTRACT}

\begin{abstract}
Aims. We present the first multi-epoch $N$-band spectro-interferometric observations of the carbon-rich Mira variable V Oph using MIDI at the ESO's Very Large Telescope Interferometer. Our aim is to study temporal variations of physical properties of the outer atmosphere and the circumstellar dust shell based on spectrally-dispersed $N$-band visibilities over the $\mathrm{C}_{2} \mathrm{H}_{2}(+\mathrm{HCN})$ features and the dust emission.

Methods. Our MIDI observations were carried out at three different phases $0.18,0.49$, and 0.65 , with three different baselines (projected baseline lengths of 42-124 m) using four $8.2 \mathrm{~m}$ Unit Telescopes (UT2-UT4, UT1-UT4, and UT2-UT3 baseline configurations). Results. The wavelength dependence of the uniform-disk diameters obtained at all epochs is characterized by a roughly constant region between 8 and $10 \mu \mathrm{m}$ with a slight dip centered at $\sim 9.5 \mu \mathrm{m}$ and a gradual increase longward of $10 \mu \mathrm{m}$. These $N$-band angular sizes are significantly larger than the estimated photospheric size of V Oph. The angular sizes observed at different epochs reveal that the object appears smaller at phase 0.49 (minimum light) with uniform-disk diameters of $\sim 5-12$ mas than at phases $0.18(\sim 12-20$ mas) and 0.65 ( $\sim 9-15$ mas). We interpret these results with a model consisting of optically thick $\mathrm{C}_{2} \mathrm{H}_{2}$ layers and an optically thin dust shell. Our modeling suggests that the $\mathrm{C}_{2} \mathrm{H}_{2}$ layers around V Oph are more extended $\left(\sim 1.7-1.8 R_{\star}\right)$ at phases 0.18 and 0.65 than at phase $0.49\left(\sim 1.4 R_{\star}\right)$ and that the $\mathrm{C}_{2} \mathrm{H}_{2}$ column densities appear to be the smallest at phase 0.49 . We also find that the dust shell consists of amorphous carbon and $\mathrm{SiC}$ with an inner radius of $\sim 2.5 R_{\star}$, and the total optical depths of $\tau_{V} \approx 0.6-0.9\left(\tau_{11.3 \mu \mathrm{m}} \approx 0.003\right.$ and 0.004 for amorphous carbon and $\mathrm{SiC}$, respectively) found at phases 0.18 and 0.65 are higher than the value obtained at phase $0.49, \tau_{V} \approx 0.3\left(\tau_{11.3 \mu \mathrm{m}} \approx 0.001\right.$ and 0.002 for amorphous carbon and $\mathrm{SiC}$, respectively).

Conclusions. Our MIDI observations and modeling indicate that carbon-rich Miras also have extended layers of polyatomic molecules as previously confirmed in oxygen-rich Miras. The temporal variation of the $\mathrm{N}$-band angular size is largely governed by the variations of the opacity and the geometrical extension of the $\mathrm{C}_{2} \mathrm{H}_{2}$ layers and the dust shell, and consequently, this masks the size variation of the photosphere. Also, the observed weakness of the mid-infrared $\mathrm{C}_{2} \mathrm{H}_{2}$ absorption in carbon-rich Miras can be explained by the emission from the extended $\mathrm{C}_{2} \mathrm{H}_{2}$ layers and the dust shell.
\end{abstract}

Key words. infrared: stars - techniques: interferometric - stars: circumstellar matter - stars: carbon - stars: AGB and post-AGB stars: individual: V Oph

\section{Introduction}

The driving mechanism of mass outflows in Mira variables has not yet been fully understood. Recent progress in optical and infrared interferometric techniques has been contributing to studies of the region between the top of the photosphere and the innermost region of the circumstellar dust shell, exactly where mass outflows are expected to be initiated. Near-infrared interferometric observations of several oxygen-rich and S-type $(\mathrm{C} / \mathrm{O} \approx 1)$ Mira variables (Mennesson et al. 2002; Perrin et al. 2004; Ireland et al. 2004; Woodruff et al. 2004; Fedele et al. 2005) have turned out to be consistent with the presence of dense molecular layers extending to $\sim 2 R_{\star}$, which were introduced

* Based on observations made with the Very Large Telescope Interferometer of the European Southern Observatory. Program ID: 075.D-0607.

$\star \star$ Table 4 is only available in electronic form at the CDS via anonymous ftp to cdsarc.u-strasbg.fr (130.79.128.5) or via http://cdsweb.u-strasbg.fr/cgi-bin/qcat?]/A+A/466/1099 to explain spectroscopic observations (e.g., Hinkle et al. 1979; Tsuji et al. 1997; Yamamura et al. 1999). Furthermore, based on semi-empirical models, Ohnaka (2004a) quantitatively shows that near-infrared ( $K$ and $L^{\prime}$ bands) and mid-infrared $(11 \mu \mathrm{m})$ interferometric and spectroscopic observations of three oxygenrich and S-type Miras ( $o$ Cet, R Leo, and $\chi$ Cyg) can be explained by dense warm water vapor layers extending to $\sim 2.5 R_{\star}$ with temperatures of $1200-2000 \mathrm{~K}$ and $\mathrm{H}_{2} \mathrm{O}$ column densities of the order of $10^{21} \mathrm{~cm}^{-2}$. These dense water vapor layers are responsible for the increase of the angular size from the near-infrared to the mid-infrared observed toward oxygen-rich Miras.

However, these studies have been limited to oxygen-rich or S-type Mira variables up to now, and the physical properties of the outer atmosphere of carbon stars and their temporal variations have not yet been well probed. Observations of carbonrich Miras as well as non-Mira carbon stars using the Short Wavelength Spectrometer (SWS) onboard the Infrared Space Observatory (ISO) revealed that while the spectra of carbon stars shortward of $\sim 5 \mu \mathrm{m}$ can be fairly reproduced by non-gray 
hydrostatic or dynamical model atmospheres, these models entirely fail to explain the observed spectra longward of $\sim 5 \mu \mathrm{m}$, as demonstrated by Jørgensen et al. (2000) and Gautchy-Loidl et al. (2004). These models predict very strong absorption due to $\mathrm{C}_{2} \mathrm{H}_{2}$ and $\mathrm{HCN}$ at 7 and $14 \mu \mathrm{m}$, but the observed ISO spectra of carbon stars show only weak absorption due to these molecular species. As a possible solution for this discrepancy, GautchyLoidl et al. (2004) show that dynamical models with dust-driven stellar winds can provide a qualitative explanation for the absence of strong absorption features due to $\mathrm{C}_{2} \mathrm{H}_{2}$ and $\mathrm{HCN}$ at least for stars with mass loss rates of $\sim 10^{-6} M_{\odot} \mathrm{yr}^{-1}$, although these models cannot yet reproduce the observed strengths of the molecular features quantitatively. On the other hand, Aoki et al. $(1998,1999)$ propose that emission from extended warm molecular layers containing $\mathrm{C}_{2} \mathrm{H}_{2}$ and $\mathrm{HCN}$ may be responsible for the discrepancy between the ISO spectra and the photospheric models.

Infrared interferometry provides us with an excellent opportunity to probe the nature of this controversial region - the outer atmosphere and the innermost region of the dust shell of carbon stars. Van Belle et al. (1997) observed two carbon-rich Miras U Cyg and V Cyg at 2-3 epochs in the $K$ band using the Infrared Optical Telescope Array (IOTA), but the accuracy of the derived angular sizes as well as the presence of molecular absorption, which affects the apparent stellar size in these broadband observations, makes the deduction of temporal variations difficult. Thompson et al. (2002) brought about a clearer picture of the phase dependence of the angular size of the carbon-rich Mira RZ Peg based on multi-epoch narrow-band observations in the $K$-band using the Palomar Testbed Interferometer (PTI): the uniform-disk diameters measured over two pulsational cycles show a variation from 2.3 to 3.0 mas. Their measurements also show that the observed uniform-disk diameter monotonically increases from 2.0 to $2.4 \mu \mathrm{m}$, which can be interpreted as that the strong CO first overtone bands longward of $2.3 \mu \mathrm{m}$ make the star appear larger than at shorter wavelengths where only relatively weak (but crowded) $\mathrm{CN}$ lines are present.

However, mid-infrared interferometric observations of carbon stars (particularly, not very dusty ones) are still very scarce, as are multi-epoch measurements to follow temporal variations of the physical properties of the photosphere and the circumstellar environment. The MID-infrared Interferometric instrument (MIDI) at the ESO's Very Large Telescope Interferometer (VLTI) is well suited for a study of the circumstellar environment close to the star. It enables us to obtain visibilities with a spectral resolution of 30 or 230 from 8 to $13 \mu \mathrm{m}$, where the absorption features due to $\mathrm{C}_{2} \mathrm{H}_{2}$ and $\mathrm{HCN}$ as well as the $\mathrm{SiC}$ and (featureless) amorphous carbon dust emission are observed for carbon stars.

In this paper, we present the first multi-epoch $N$-band spectro-interferometric observations of the carbon-rich Mira star V Oph using MIDI. Our object V Oph has a period of 297 days (Samus et al. 2004), and the $V$ magnitude varies from 7.3 to 11.6 $\left(F_{12 \mu \mathrm{m}} \approx 20 \mathrm{Jy}\right)$. Its spectral energy distribution (SED) suggests that the circumstellar dust shell is optically thin with quite a low mass loss rate of $\sim 10^{-8} M_{\odot} \mathrm{yr}^{-1}$ (Groenewegen et al. 1998). The absence of a thick dust shell makes V Oph suitable for probing the presence or absence of extended molecular layers in carbonrich Miras.

\section{MIDI observations}

V Oph was observed with MIDI on six nights between April and September 2005 in service mode (Program ID: 075-D0607,
Table 1. Summary of MIDI observations of V Oph: night, time of observation $(\mathrm{UTC}=$ Coordinated Universal Time), airmass (AM), telescope configurations (Tel., UT=Unit Telescope), projected baseline length $B_{\mathrm{p}}$, position angle of the baseline vector on the sky (PA), and variability phase estimated from the visual light curve compiled by the American Association of Variable Star Observers (AAVSO).

\begin{tabular}{cccccccc}
\hline \hline$\#$ & $\begin{array}{c}\text { Night } \\
(2005)\end{array}$ & $\begin{array}{c}t_{\text {obs }} \\
(\text { UTC) }\end{array}$ & AM & Tel. & $\begin{array}{c}B_{\mathrm{p}} \\
(\mathrm{m})\end{array}$ & $\begin{array}{r}\text { PA } \\
\left({ }^{\circ}\right)\end{array}$ & Phase \\
\hline 1 & Apr. 17 & $08: 39: 02$ & 1.1 & UT2-4 & 85.6 & 85.4 & 0.18 \\
2 & Apr. 18 & $04: 51: 38$ & 1.3 & UT2-4 & 71.8 & 70.1 & 0.18 \\
3 & Jul. 19 & $03: 03: 08$ & 1.1 & UT1-4 & 124.2 & 65.6 & 0.49 \\
4 & Jul. 23 & $22: 58: 21$ & 1.2 & UT2-3 & 41.9 & 25.7 & 0.49 \\
5 & Aug. 24 & $23: 49: 30$ & 1.1 & UT2-4 & 87.6 & 84.3 & 0.61 \\
6 & Sep. 18 & $23: 27: 32$ & 1.2 & UT1-4 & 120.0 & 66.0 & 0.69 \\
\hline
\end{tabular}

P.I.: K. Ohnaka), as summarized in Table 1. A prism with a spectral resolution of $\lambda / \Delta \lambda \simeq 30$ at $10 \mu \mathrm{m}$ was used to obtain spectrally dispersed fringes between 8 and $13 \mu \mathrm{m}$. A detailed description of the observing procedure is given in Przygodda et al. (2003), Leinert et al. (2004), and Chesneau et al. (2005a,b).

We use two different MIDI data reduction packages: MIA developed at the Max-Planck-Institut für Astronomie and EWS developed at the Leiden Observatory (MIA+EWS, ver.1.4) ${ }^{1}$. While the MIA package is based on the power spectrum analysis, which measures the total power of observed fringes (Leinert et al. 2004), the EWS software first corrects for optical path differences (instrumental as well as atmospheric delays) in each scan, and then, the fringes are coherently added (Jaffe 2004). We derive the interferometer transfer function at each wavelength (i.e., at each spectral channel) between 8 and $13 \mu \mathrm{m}$ by observing calibrators whose angular diameters are known (see Table 2). In order to calibrate the visibility of V Oph at a given wavelength, we use the mean of the transfer function values derived from all the calibrators observed on the same night as V Oph, and the errors of the calibrated visibilities are estimated from the standard deviation of the transfer function values at each wavelength. The results obtained with the MIA and EWS packages show good agreement, and we only present the result reduced with the EWS package below. The relative errors of the calibrated visibilities are typically $\pm 10-15 \%(1 \sigma)$, and the error sources are described in Ohnaka et al. (2005).

Figure 1 shows the calibrated visibilities of V Oph obtained on six nights (the complete set of the observed visibilities are electronically available in Table 4 ). The observed $N$-band visibilities except for the data \#4 taken with the shortest baseline on 2005 July 23 are characterized by a monotonic increase from 8 to $\sim 9.5 \mu \mathrm{m}$ and a gradual decrease longward of $\sim 10 \mu \mathrm{m}$. Figures $2 \mathrm{a}-\mathrm{c}$ show the observed visibilities plotted as a function of spatial frequency at three representative wavelengths between 8 and $13 \mu \mathrm{m}$. In the modeling described below, the data sets are binned into three epochs: epoch 1 (2005 Apr. $17+18$, phase 0.18), epoch 2 (2005 Jul. $19+23$, phase 0.49), and epoch 3 (2005 Aug. $24+$ Sep. 18 , phase $0.61-0.69$ with an average of $0.65)$. The data sets \#1 and \#5, which were obtained four months apart but at almost the same baseline lengths and position angles, reveal a temporal variation of visibility, particularly at $8.3 \mu \mathrm{m}$. However, the errors of the measured visibilities become larger at longer wavelengths, which makes the detection of temporal variations less conclusive. For example, the large errors of the

1 Available at http://Www.mpia-hd.mpg.de/MIDISOFT/ and http://www.strw. leidenuniv.nl/ nevec/MIDI/index.html 

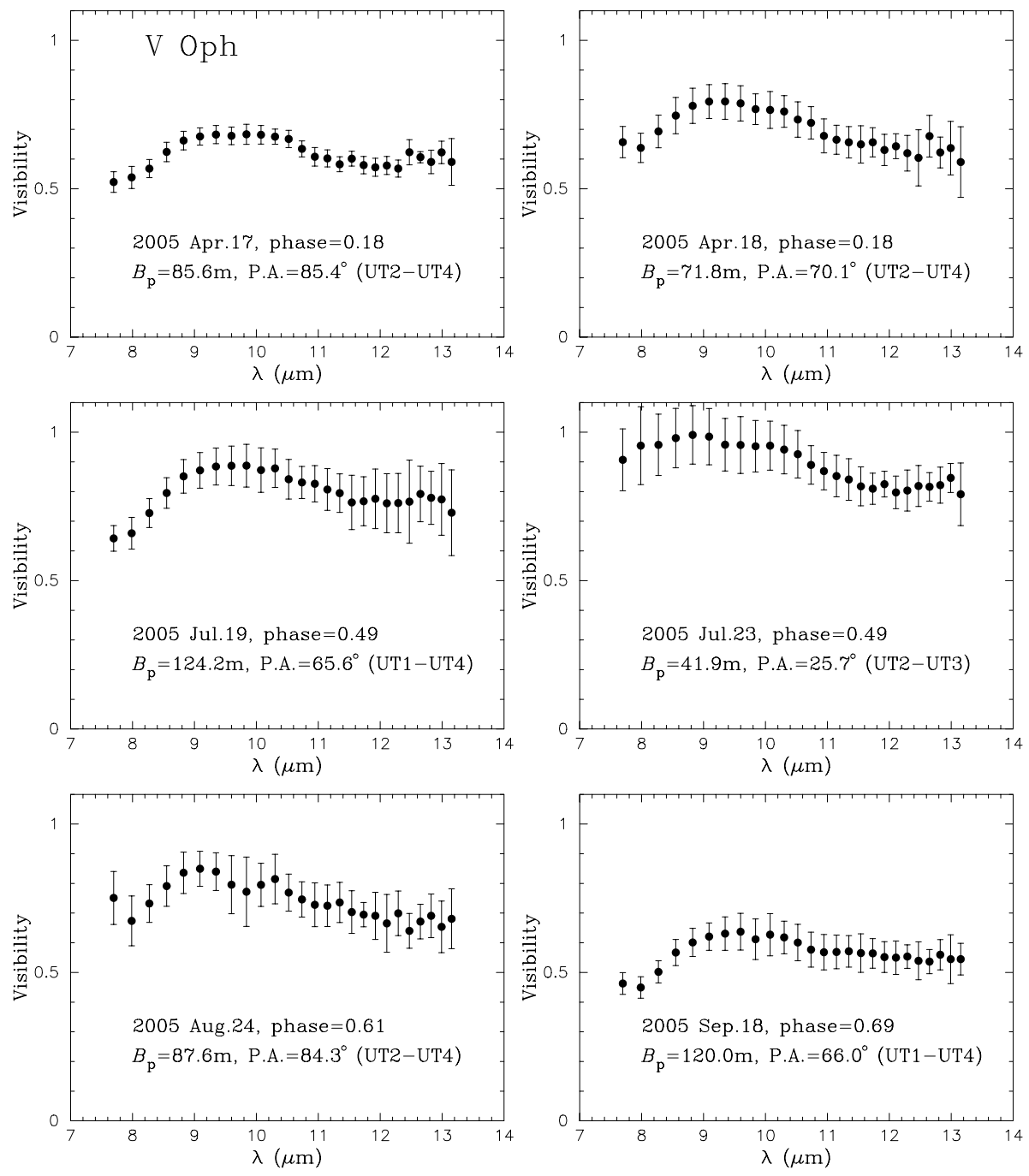

Fig. 1. $N$-band visibilities of $\mathrm{V}$ Oph observed with MIDI on six nights between April and September 2005.

visibilities measured at $12.5 \mu \mathrm{m}$ hamper us from definitively detecting a temporal variation. On the other hand, the data sets \#3 and \#6, which were obtained two months apart at approximately the same baseline lengths and position angles, clearly indicate a temporal variation not only at $8.3 \mu \mathrm{m}$ but also at 10 and $12.5 \mu \mathrm{m}$.

At each epoch, we fit the visibilities at a given wavelength with a uniform disk as shown in Figs. $2 \mathrm{a}-\mathrm{c}$. While the differences in position angle for the data sets of the first and third epochs are not very large (they differ by $15.3^{\circ}$ and $18.3^{\circ}$, respectively), the data sets of the second epoch (\#3 and \#4) were obtained with quite different position angles of $65.6^{\circ}$ and $25.7^{\circ}$. We tentatively fit the visibility points of these data sets with a single uniform disk, regardless the position angles, because the difference in baseline length between two data sets makes it impossible to draw a conclusion about the possible presence or absence of asymmetries. The resulting uniform-disk diameters derived for three epochs are plotted as a function of wavelength in Fig. 2d, which illustrates a temporal variation of the angular size of V Oph: the star appears smaller at minimum light (phase 0.49) than at post-maximum (phase 0.18) or postminimum (phase 0.65). It should be stressed, however, that we use uniform-disk fits to obtain some kind of representative angular size of the object and that it can be very misleading to deduce quantitatively temporal variations from these uniformdisk diameters, particularly if the real intensity distribution of the object is very different from a uniform disk.
The photospheric angular size of V Oph (i.e., the stellar angular diameter measured in the continuum) has not been directly measured, but we can estimate it from the bolometric flux and effective temperature, if the radiation is assumed to be isotropic. The bolometric flux of $\mathrm{V}$ Oph near maximum light is derived to be $4.4 \times 10^{-10} \mathrm{~W} \mathrm{~m}^{-2}$ from photometric data available in the literature, while the effective temperature is estimated to be 2600-3000 K (see Sect. 3 for discussion on photometric data and effective temperature). These bolometric flux and effective temperatures translate into a photospheric angular size of 4-6 mas. It means that the $N$-band angular sizes obtained at three epochs are significantly larger than the photospheric size in most cases. Only the $9-10 \mu \mathrm{m}$ uniform-disk diameters obtained at phase 0.49 are rather close to the photospheric size. Therefore, for the carbon-rich Mira V Oph, we detect the same trend as found in oxygen-rich Mira stars: the mid-infrared angular sizes are much larger than the photospheric angular size.

We also extract the absolutely calibrated $N$-band spectra of $\mathrm{V}$ Oph from the MIDI data by dividing the observed spectra of V Oph by that of a spectrophotometric standard star and then multiplying the absolutely calibrated spectrum of this latter standard star. Interferometric calibrators observed close in time to $\mathrm{V}$ Oph at similar airmasses are used to extract the spectra of $\mathrm{V}$ Oph as marked with $\dagger$ in Table 2 . The absolutely calibrated spectra of these spectrophotometric standard stars are taken from Cohen et al. (1999) if available. Otherwise, the absolutely 
Table 2. List of calibrators used for the calibration of the V Oph data, together with $12 \mu \mathrm{m}$ fluxes $\left(F_{12}\right)$, uniform-disk diameters $\left(d_{\mathrm{UD}}\right)$, the date as well as the time stamp $\left(t_{\mathrm{obs}}\right)$ of MIDI observations, and airmass (AM). The uniform-disk diameters were taken from the CalVin list available at ESO (http://www.eso.org/observing/etc/). The data sets used for spectrophotometric calibration of the spectra of V Oph are marked with $\dagger$.

\begin{tabular}{|c|c|c|c|c|c|}
\hline Calibrator & $\begin{array}{l}F_{12} \\
(\mathrm{Jy})\end{array}$ & $\begin{array}{c}d_{\mathrm{UD}} \\
(\mathrm{mas})\end{array}$ & $\begin{array}{c}\text { Night } \\
(2005)\end{array}$ & $\begin{array}{l}t_{\mathrm{obs}} \\
\text { (UTC) }\end{array}$ & $\overline{\overline{\mathrm{AM}}}$ \\
\hline HD120404 & 13.3 & $3.03 \pm 0.24$ & Apr. 17 & $02: 48: 03$ & 1.5 \\
\hline \multirow[t]{2}{*}{ HD142804 } & 11.7 & $2.80 \pm 0.08$ & Apr. 17 & $04: 21: 08$ & 1.2 \\
\hline & & & Apr. 18 & $03: 26: 25$ & 1.5 \\
\hline \multirow[t]{3}{*}{ HD169767 } & 8.8 & $2.16 \pm 0.11$ & Apr. 17 & $06: 08: 59$ & 1.4 \\
\hline & & & Apr. 17 & 07:18:00 & 1.2 \\
\hline & & & Apr. 17 & $07: 53: 19$ & 1.2 \\
\hline \multirow[t]{6}{*}{ HD150052 } & 8.9 & $2.40 \pm 0.07$ & Apr. 17 & $08: 17: 33^{\dagger}$ & 1.1 \\
\hline & & & Apr. 18 & 04:29:57 & 1.4 \\
\hline & & & Jul. 19 & $02: 43: 38^{\dagger}$ & 1.1 \\
\hline & & & Jul. 23 & $23: 20: 17$ & 1.2 \\
\hline & & & Aug. 24 & $23: 22: 48^{\dagger}$ & 1.0 \\
\hline & & & Sep. 18 & $23: 51: 54^{\dagger}$ & 1.3 \\
\hline \multirow[t]{4}{*}{ HD188512 } & 9.0 & $2.07 \pm 0.10$ & Apr. 17 & 09:04:41 & 1.3 \\
\hline & & & Jul. 23 & 02:58:55 & 1.3 \\
\hline & & & Jul. 23 & 04:03:14 & 1.2 \\
\hline & & & Jul. 23 & $06: 37: 58$ & 1.4 \\
\hline \multirow[t]{5}{*}{ HD169916 } & 31.2 & $3.90 \pm 0.21$ & Apr. 17 & $10: 08: 16^{\dagger}$ & 1.0 \\
\hline & & & Apr. 18 & 07:24:08 & 1.1 \\
\hline & & & Jul. 19 & $05: 46: 15$ & 1.2 \\
\hline & & & Jul. 23 & $00: 04: 56^{\dagger}$ & 1.3 \\
\hline & & & Jul. 23 & $05: 49: 35$ & 1.3 \\
\hline HD37160 & 9.4 & $2.08 \pm 0.20$ & Apr. 18 & $23: 26: 37$ & 1.7 \\
\hline HD122451 & 11.4 & $0.96 \pm 0.07$ & Apr. 18 & $02: 26: 48$ & 1.4 \\
\hline \multirow[t]{2}{*}{ HD152885 } & 12.6 & $2.88 \pm 0.09$ & Apr. 18 & $05: 12: 20$ & 1.2 \\
\hline & & & Jul. 23 & 00:48:11 & 1.0 \\
\hline \multirow[t]{3}{*}{ HD157236 } & 10.5 & $2.58 \pm 0.14$ & Apr. 18 & $06: 21: 08^{\dagger}$ & 1.1 \\
\hline & & & Apr. 18 & $08: 16: 35$ & 1.0 \\
\hline & & & Aug. 24 & 01:40:04 & 1.1 \\
\hline HD160668 & 8.4 & $2.30 \pm 0.10$ & Apr. 18 & 06:43:00 & 1.1 \\
\hline HD168723 & 17.3 & $2.88 \pm 0.13$ & Apr. 18 & $09: 17: 26$ & 1.1 \\
\hline \multirow[t]{4}{*}{ HD152820 } & 10.9 & $2.63 \pm 0.16$ & Jul. 19 & $04: 14: 50$ & 1.2 \\
\hline & & & Jul. 23 & $02: 15: 18$ & 1.0 \\
\hline & & & Jul. 23 & 05:01:41 & 1.5 \\
\hline & & & Sep. 18 & $00: 40: 11^{\dagger}$ & 1.3 \\
\hline HD178345 & 12.5 & $2.50 \pm 0.07$ & Aug. 24 & $04: 24: 35$ & 1.3 \\
\hline HD165135 & 23.4 & $3.38 \pm 0.16$ & Sep. 18 & $01: 30: 20$ & 1.2 \\
\hline
\end{tabular}

calibrated spectrum of a standard star is calculated as follows. First, we select a star in Cohen et al. (1999) with the same spectral type and luminosity class as our standard star. Then the flux level of the spectrum of this "template" star is scaled using its angular diameter given in Cohen et al. (1999) and that of our standard star given in Table 2, and the resulting spectrum is used for spectrophotometric calibration. Finally, we average all the resulting spectra obtained at each epoch. The absolutely calibrated spectra of V Oph observed at three epochs are shown in Fig. 3. The errors of the MIDI spectra are difficult to estimate properly from the present data sets, because the number of standard stars observed close in time to $\mathrm{V}$ Oph and at similar airmasses is quite limited $(3,2$, and 3 standard stars at epoch 1,2 , and 3, respectively). However, we estimate the errors of the absolutely calibrated spectra to be $10-20 \%$, based on other studies which derived $N$-band spectra from MIDI data (e.g., Kervella et al. 2006; Poncelet et al. 2006; Quanz et al. 2006).
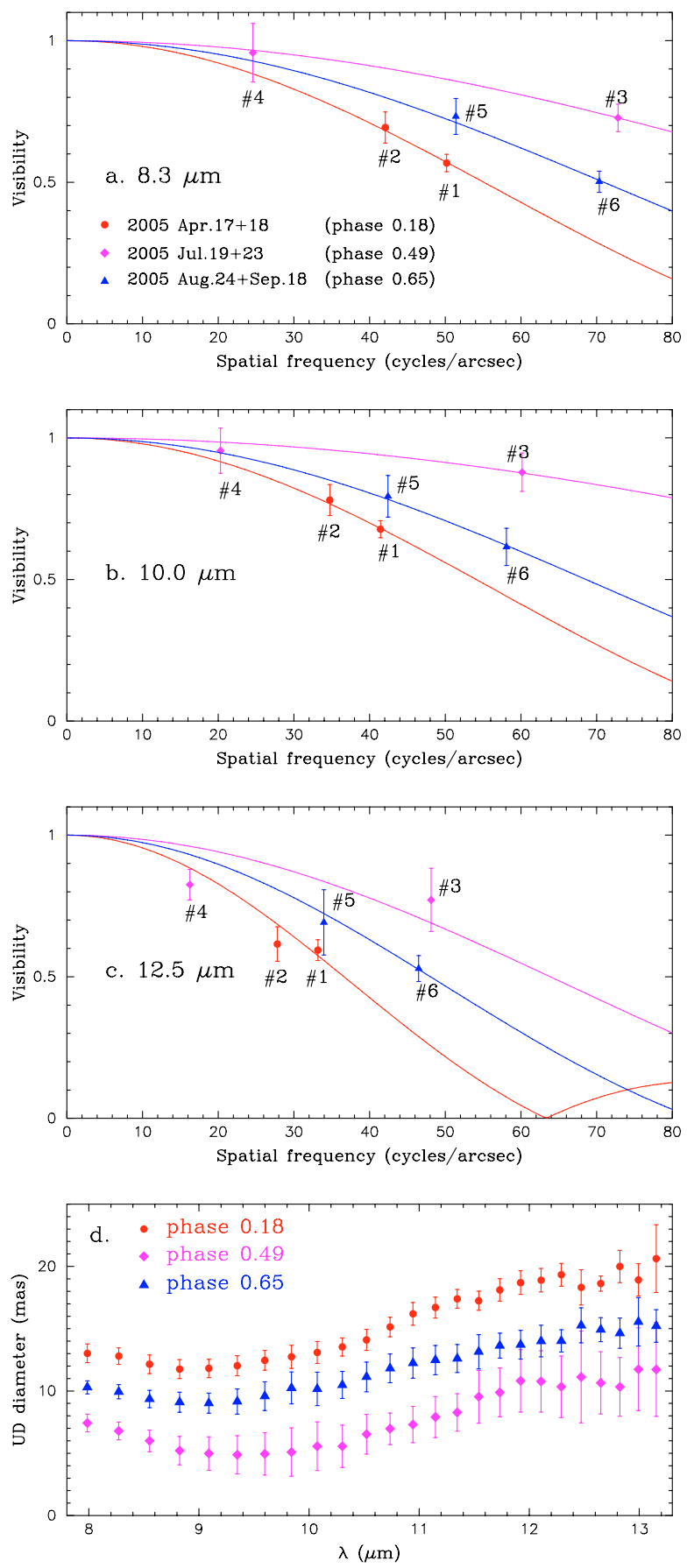

Fig. 2. $N$-band visibilities of V Oph plotted as a function of spatial frequency at three representative wavelengths. a) $8.3 \mu \mathrm{m}$, b) $10.0 \mu \mathrm{m}$, and c) $12.5 \mu \mathrm{m}$. In these panels, the filled circles, diamonds, and triangles represent the data obtained at epoch 1 (phase 0.18), epoch 2 (phase 0.49), and epoch 3 (phase 0.65), respectively, as defined in Sect. 2. The fit with uniform disks is also plotted for three epochs. The data set number of each visibility point as given in Table 1 is indicated. d) Uniform-disk (UD) diameters derived by fitting the visibility points obtained at each epoch are plotted as a function of wavelength. The filled circles, diamonds, and triangles represent the data obtained at epoch 1,2 , and 3 , respectively.

Figure 3 reveals that the spectra are characterized by absorption between 8 and $9 \mu \mathrm{m}$ and a broad emission feature centered at $\sim 11.3 \mu \mathrm{m}$. The feature seen between 8 and $9 \mu \mathrm{m}$ is actually the tail of the broad absorption feature centered at $\sim 7.5 \mu \mathrm{m}$ due to the $\mathrm{C}_{2} \mathrm{H}_{2} v_{4}+v_{5}$ band and the HCN $2 v_{2}$ band. However, as shown 


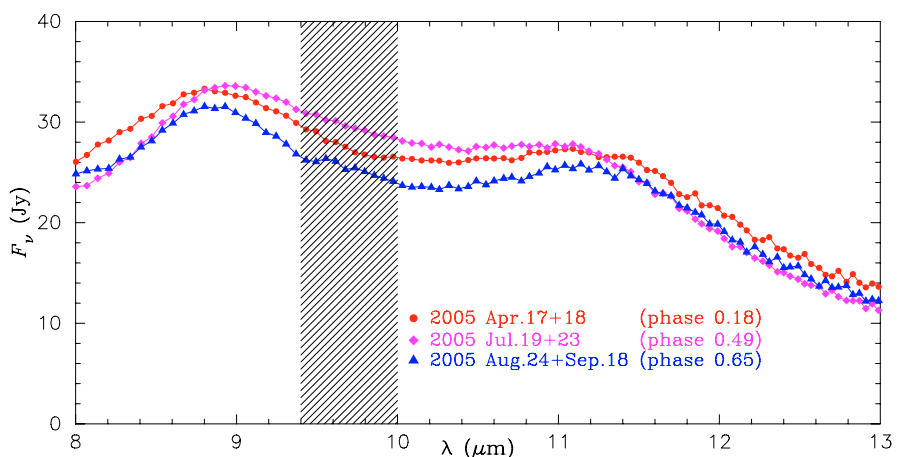

Fig. 3. $N$-band spectra of V Oph obtained at three epochs. The filled circles, diamonds, and triangles represent the spectra obtained at epochs 1,2 , and 3 , respectively. The shaded region represents the wavelength range severely affected by the telluric ozone absorption centered at $9.6 \mu \mathrm{m}$.

in Sect. 4.1, the HCN $2 v_{2}$ band extends only up to $\sim 8.3 \mu \mathrm{m}$, which leaves $\mathrm{C}_{2} \mathrm{H}_{2}$ as the dominant opacity source between 8 and $9 \mu \mathrm{m}$. The broad emission feature at $\sim 11.3 \mu \mathrm{m}$ is attributed to $\mathrm{SiC}$ dust. In addition to these conspicuous features within the $N$ band, a broad absorption feature centered at $\sim 14 \mu \mathrm{m}$ due to the $\mathrm{C}_{2} \mathrm{H}_{2} v_{5}$ and $\mathrm{HCN} v_{2}$ bands is identified in ISO spectra of other carbon-rich Mira variables (e.g., Yamamura et al. 1997; Hron et al. 1998; Aoki et al. 1999). This absorption feature extends down to $\sim 11 \mu \mathrm{m}$, where it becomes blended with the $\mathrm{SiC}$ emission feature. Comparison between the spectra at phases 0.18 and 0.49 reveals that the $8 \mu \mathrm{m}$ absorption feature becomes somewhat stronger at minimum light than at post-maximum. This trend is similar to that found in the Mira-like carbon star R Scl by Hron et al. (1998). We will discuss this variation of the $8 \mu \mathrm{m}$ feature in Sect. 4.2 .

\section{Modeling with a dust shell}

As briefly discussed in Sect. 2, the $N$-band uniform-disk diameters measured with MIDI are remarkably larger than the estimated photospheric angular size, which suggests the contribution of circumstellar material in the mid-infrared angular size. This contribution can be of molecular and/or dust origin. We first attempt to interpret the observed $N$-band visibilities and spectra with dust shell models.

We calculate spherically symmetric dust shell models, using the multi-dimensional Monte Carlo code mcsim_mpi (Ohnaka et al. 2006a), which computes dust temperatures in radiative equilibrium and generates model SEDs. Then the model image at each wavelength within the $N$ band is produced with the ray-trace method as described in Ohnaka et al. (2006a), and the corresponding monochromatic visibility is calculated by taking the Hankel transform of the model image. The monochromatic visibilities are then spectrally convolved to a resolution of 30 to match the MIDI observations. The effective temperature of V Oph derived by various authors ranges from 2650 to $3010 \mathrm{~K}$ (Ohnaka \& Tsuji 1996; Bergeat et al. 2001; Groenewegen 2006), but the phase dependence of effective temperature of $\mathrm{V} O \mathrm{Oph}$ is not well established. Therefore, in the dust shell modeling below, we adopt a fixed effective temperature of $2800 \mathrm{~K}$, which is roughly the mean of the above range, and the central star is approximated with the blackbody of $2800 \mathrm{~K}$. We assume that the dust shell consists of amorphous carbon and $\mathrm{SiC}$, following the results of Groenewegen et al. (1998) and Groenewegen (2006), who concluded that the dust chemical composition around $\mathrm{V}$ Oph is dominated by amorphous carbon $(85 \%)$ with a minor contribution of $\mathrm{SiC}(15 \%)$. We compute a grid of models with the following parameter ranges: $r_{\text {in }}\left(R_{\star}\right)=$ $2.5 \ldots 5.0\left(\Delta r_{\text {in }}=0.5\right), \tau_{V}^{\text {amc }}=0.2 \ldots 0.8\left(\Delta \tau_{V}^{\text {amc }}=0.2\right)$, where $r_{\text {in }}$ and $\tau_{V}^{\text {amc }}$ denote the inner boundary radius (assumed to be the same for amorphous carbon and $\mathrm{SiC}$ ) and the optical depth of amorphous carbon dust at $0.55 \mu \mathrm{m}$, respectively. The relative contribution of $\mathrm{SiC}, \tau_{V}^{\mathrm{SiC}} /\left(\tau_{V}^{\mathrm{amc}}+\tau_{V}^{\mathrm{SiC}}\right)$ is varied from 0.1 to 0.4 with an increment of 0.1 , where $\tau_{V}^{\mathrm{SiC}}$ denotes the optical depth of $\mathrm{SiC}$ dust at $0.55 \mu \mathrm{m}$. The opacities of amorphous carbon and $\mathrm{SiC}$ are calculated from complex refractive indices presented by Rouleau \& Martin (1991, AC1 sample) and by Pégourié (1988), respectively, assuming a single grain size of $0.1 \mu \mathrm{m}$ for both species. The grain density distribution is assumed to be proportional to $r^{-2}$, and the outer radius of the shell is fixed to $10^{3} \times r_{\mathrm{in}}$.

Figure 4 shows a comparison between the observational data and a model which can well reproduce the SED observed near maximum light. We derived the observed SED from optical and infrared photometric data available in the literature (Landolt 1968, 1973; Walker 1979; Noguchi et al. 1981; Whitelock et al. 2000; IRAS Point Source Catalog) and dereddened with $A_{V}=$ 0.6 (Groenewegen et al. 1998) using the method of Savage \& Mathis (1979) with $A_{V}=3.1 E(B-V)$. Unfortunately, most of the photometric data, particularly in the optical, were obtained at phases near maximum light, and therefore, we can compare model SEDs with the observed one only for phase 0.18. As can be seen in Figs. $4 a$ and $4 b$, the SED at maximum light as well as the MIDI spectrum obtained at phase 0.18 can be explained by the model with $r_{\text {in }}=2.5 R_{\star}, \tau_{V}^{\text {amc }}=0.6(0.0038$ at $11.3 \mu \mathrm{m})$, and $\tau_{V}^{\mathrm{SiC}}=0.26(0.0078$ at $11.3 \mu \mathrm{m})$. The temperatures of amorphous carbon and $\mathrm{SiC}$ dust at the inner boundary are $\sim 1580 \mathrm{~K}$, which roughly corresponds to the condensation temperature of amorphous carbon predicted by dynamical models (e.g., Nowotny et al. 2005a) and is in agreement with the value that Groenewegen et al. (1998) derived from a SEDfitting (note, however, that condensation temperatures generally depend on detailed grain nucleation and growth processes). The model cannot reproduce the $8 \mu \mathrm{m}$ feature, because the star is approximated with a blackbody without a detailed model atmosphere. This is also the reason why the model overestimates the flux in the optical where strong molecular absorption is present. Comparison of the visibilities shown in Fig. 4c reveals that the dust shell model can roughly reproduce the observed visibility levels. The dip at $\sim 11.3 \mu \mathrm{m}$ seen in the model visibilities corresponds to the position of the $\mathrm{SiC}$ emission feature. The flux contribution of the dust shell becomes larger due to the SiC emission feature at these wavelengths, which leads to lower visibilities. It should be noted that the scaling factor between a model SED and the observed one is given by $\left(R_{\star} / d\right)^{2}$, where $d$ is the distance of $\mathrm{V}$ Oph. The model visibilities are scaled with the angular radius of the central star $\left(R_{\star} / d\right)$ derived by fitting the model SEDs to the observed data.

However, there are noticeable discrepancies between the model visibilities and the MIDI data. Firstly, the models cannot reproduce the observed wavelength dependence of visibility between 8 and $9 \mu \mathrm{m}$ as well as longward of $12 \mu \mathrm{m}$, where the $\mathrm{C}_{2} \mathrm{H}_{2}$ (and $\mathrm{HCN}$ ) absorption features are present, although the error bars of the observed visibilities are rather large in the latter wavelength range. The fact that the observed visibilities are smaller than the model at $8-9 \mu \mathrm{m}$ and $\gtrsim 12 \mu \mathrm{m}$ suggests that the observed angular sizes are larger than that predicted by the dust shell model. Secondly, the models cannot reproduce the shape of the observed visibilities as a function of spatial frequency, which 

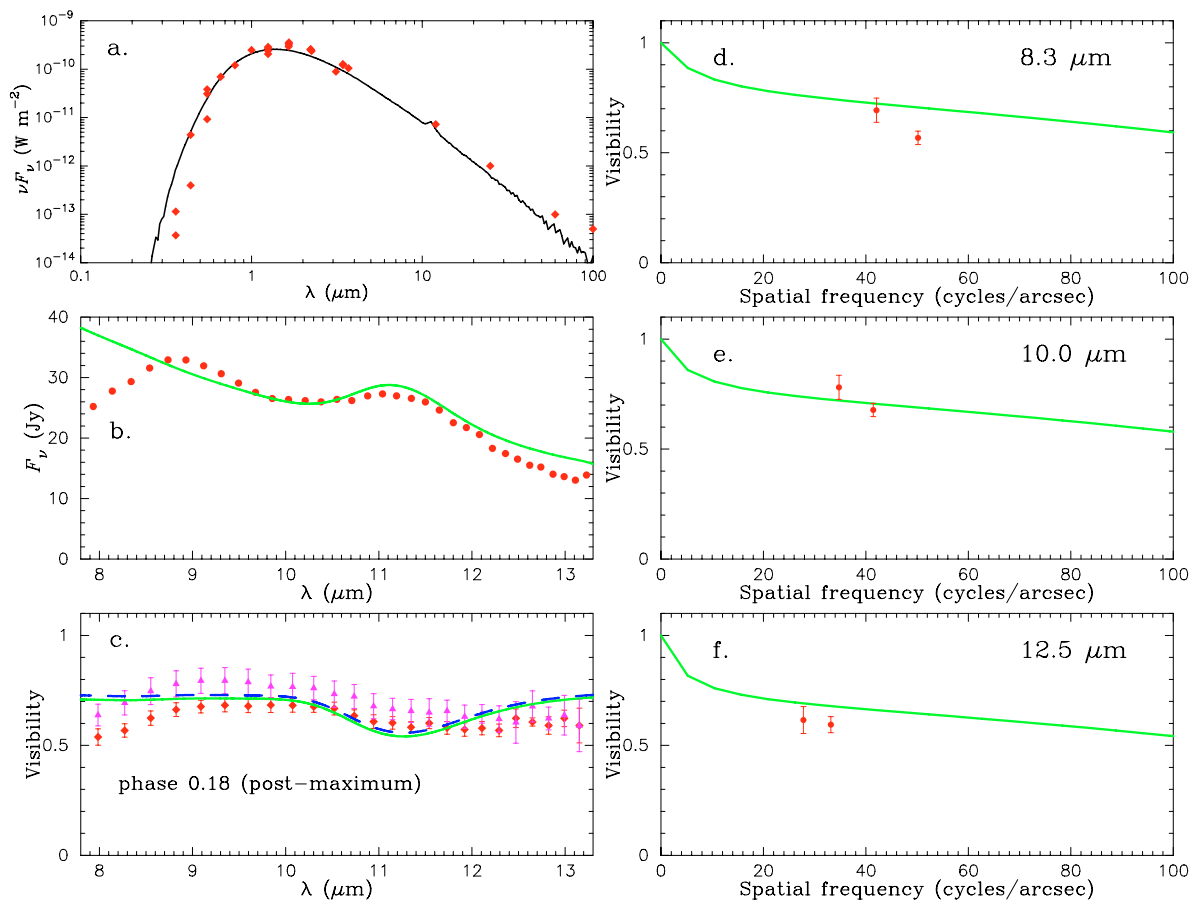

Fig. 4. Dust shell model containing amorphous carbon and $\mathrm{SiC}$ which well reproduces the observed SED for phases near maximum light. The dust shell parameters are $r_{\text {in }}=2.5 R_{\star}$, $\tau_{V}^{\mathrm{amc}}=0.6$, and $\tau_{V}^{\mathrm{SiC}}=0.26$. a) Comparison of the observed and model SEDs. The observed SEDs are represented with the filled diamonds, while the model SED is represented with the solid line. b) Comparison of the observed and model $N$-band spectra. The filled circles represent the MIDI spectrum obtained at phase 0.18 , while the solid line represents the model prediction. c) The filled diamonds and triangles represent the $N$-band visibilities measured with projected baseline lengths of 85.6 and $71.8 \mathrm{~m}$, respectively, while the solid and dashed lines represent the corresponding model visibilities. d)-f) The solid lines represent the model visibilities at $8.3,10.0$, and $12.5 \mu \mathrm{m}$ plotted as a function of spatial frequency. The observed data are plotted with the filled circles. can be seen in Fig. $4 \mathrm{~d}$ : the model visibility at $8.3 \mu \mathrm{m}$ is somewhat too flat compared to those observed. Since the dust shell is overresolved with the baselines used in our MIDI observations (the dust shell visibility component is seen as steep visibility drops at spatial frequencies from 0 to $\sim 5$ cycles/arcsec in Figs. $4 d-f$ ), the visibility shapes measured by our observations correspond to the central star component. Therefore, this disagreement of visibility shape means that the observed $N$-band angular size of the central star is larger than that used in the model. However, the angular size of the central star is derived by fitting the model SED to the observed one, and therefore, a significant change of the angular size of the central star would lead to a poorer match to the observed SED including the $N$-band spectrum. Changes in effective temperature, dust chemistry, and density gradient cannot explain the observed visibility shapes, either. Therefore, it can be postulated that there is some additional component close to the star that makes the central source appear larger than the star itself.

\section{Modeling with $\mathrm{C}_{2} \mathrm{H}_{2}$ layers and a dust shell}

As in oxygen-rich Mira variables that have dense $\mathrm{H}_{2} \mathrm{O}$ layers extending to $2-3 R_{\star}$, it is possible that similar layers consisting of polyatomic molecules exist around carbon-rich Miras, which makes the central star appear larger than its photospheric size. In carbon-rich atmospheres, $\mathrm{C}_{2} \mathrm{H}_{2}$ and $\mathrm{HCN}$ are the most abundant polyatomic species with spectral features in the $N$ band, which makes them the most likely candidates responsible for the larger apparent size of the central star component. In this section, we attempt to explain the observed data with models consisting of such molecular layers and an optically thin dust shell.

\subsection{Molecular data}

First, we describe molecular line opacity data necessary to compute models with $\mathrm{C}_{2} \mathrm{H}_{2}(+\mathrm{HCN})$ layers. In the wavelength region between 8 and $9 \mu \mathrm{m}$, the $\mathrm{C}_{2} \mathrm{H}_{2} v_{4}+v_{5}$ band is the dominant opacity source. It is also necessary to take the $\mathrm{C}_{2} \mathrm{H}_{2} v_{5}$ band centered at $14 \mu \mathrm{m}$ into account in modeling the $N$-band visibilities and spectra, because this band extends to $\sim 11 \mu \mathrm{m}$. The HCN $2 v_{2}$ band is centered at $\sim 7 \mu \mathrm{m}$, and its tail extends to $\sim 8.3 \mu \mathrm{m}$. The HCN $v_{2}$ band, which is observed together with the $\mathrm{C}_{2} \mathrm{H}_{2} v_{5}$ band in other carbon-rich Miras, is centered at $14 \mu \mathrm{m}$ and extends to $\sim 11 \mu \mathrm{m}$. Therefore, the contribution of HCN appears mainly at wavelengths between 11 and $13 \mu \mathrm{m}$, blended with the $\mathrm{C}_{2} \mathrm{H}_{2} v_{5}$ band and the $\mathrm{SiC}$ dust feature.

Despite its importance in various astrophysical environments, the line list of $\mathrm{C}_{2} \mathrm{H}_{2}$ is far from complete, particularly for high temperatures relevant to stellar photospheres and outer atmospheres (temperatures of $1000-1600 \mathrm{~K}$ are derived in our modeling below). There are a number of laboratory measurements of the molecular constants of the $v_{4}+v_{5}$ and $v_{5}$ bands, but these experiments were performed at room temperature. For example, Kabbadj et al. (1991) measured the molecular constants up to $V_{4}+V_{5} \leq 4$, which means that only 10 and 6 transitions among different $\left(V_{4}, V_{5}\right)$ are included for the $v_{5}$ and $v_{4}+v_{5}$ bands. Therefore, many hot bands expected in stellar and circumstellar environments are not included, and these lists are not appropriate for our modeling. On the other hand, Tsuji (1984) presents a totally different approach to deal with $\mathrm{C}_{2} \mathrm{H}_{2}$ opacity. He makes use of the band model method, which analytically calculates the absorption cross section at a given wavelength from basic molecular constants, instead of a detailed line list. Particularly, if the molecule is assumed to be a rigid-rotator-harmonic-oscillator (RRHO), calculations of absorption cross sections can be easily performed $^{2}$. In the present work, we use this band model opacity computed in the RRHO approximation to represent the $\mathrm{C}_{2} \mathrm{H}_{2}$ opacity, because neither laboratory measurements nor ab initio calculations at high temperatures are available yet for $\mathrm{C}_{2} \mathrm{H}_{2}$

${ }^{2}$ Figure 3 of Tsuji (1984), which shows the absorption cross section of the $\mathrm{C}_{2} \mathrm{H}_{2} \quad v_{5}$ band, has an error in the numerical labels of the ordinate. The correct range of the ordinate is from -22 to -17 instead of -23 to -18 . However, this is a mere graphical error, and the results of the calculations in Tsuji (1984) were performed with the correct cross sections (Tsuji, priv. comm.). 


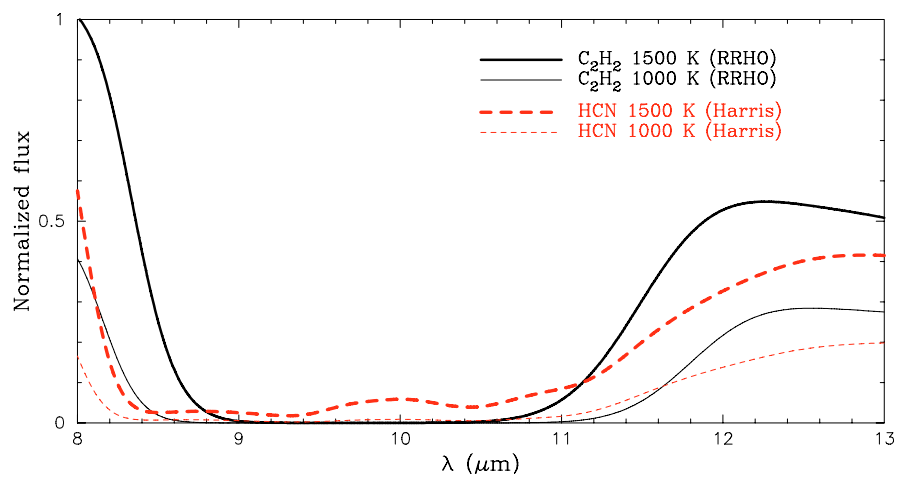

Fig. 5. Emission spectra of $\mathrm{C}_{2} \mathrm{H}_{2}$ and $\mathrm{HCN}$ from a slab of 1000 and $1500 \mathrm{~K}$. The column densities of $\mathrm{C}_{2} \mathrm{H}_{2}$ and $\mathrm{HCN}$ are set to $10^{20} \mathrm{~cm}^{-2}$. The spectra are convolved with a spectral resolution of 30 . The opacity of $\mathrm{C}_{2} \mathrm{H}_{2}$ is computed with the band model method as described in Sect. 4.1, while that of $\mathrm{HCN}$ is computed from the line list of Harris et al. (2002).

lines, and individual lines are not resolved with MIDI's spectral resolution of 30 .

Compared to $\mathrm{C}_{2} \mathrm{H}_{2}$, studies on $\mathrm{HCN}$ line lists including high vibrational levels are more advanced. Harris et al. (2002) have calculated a new, extensive ab initio line list of HCN/HNC including energy levels up to $18000 \mathrm{~cm}^{-1}$. We use their line list to calculate the HCN opacity in the modeling below. Figure 5 shows emission spectra of $\mathrm{C}_{2} \mathrm{H}_{2}$ and $\mathrm{HCN}$ from a slab of 1000 and $1500 \mathrm{~K}$ with the column densities of both molecules set to $10^{20} \mathrm{~cm}^{-2}$. The HCN opacity was calculated assuming local thermodynamical equilibrium (LTE) adopting a Gaussian profile with a $F W H M$ of $5 \mathrm{~km} \mathrm{~s}^{-1}$, and the opacity of $\mathrm{C}_{2} \mathrm{H}_{2}$ was calculated with the band model method with the RRHO approximation, which also implicitly assumes LTE. The spectra are convolved with a spectral resolution of 30 as used in the MIDI observations. The figure illustrates that $\mathrm{C}_{2} \mathrm{H}_{2}$ is the dominant opacity source at wavelengths between 8 and $9 \mu \mathrm{m}$ as well as longward of $11 \mu \mathrm{m}$, while the contribution of HCN appears primarily at wavelengths longer than $11 \mu \mathrm{m}$ (and at the shorter edge of the $N$ band). Since this contribution of $\mathrm{HCN}$ is blended with the $\mathrm{C}_{2} \mathrm{H}_{2}$ $v_{5}$ band and the SiC dust feature, the effects of $\mathrm{HCN}$ on spectra and visibilities tend to be masked by the $\mathrm{C}_{2} \mathrm{H}_{2}$ and $\mathrm{SiC}$ features, which hinders us from putting strong constraints on the $\mathrm{HCN}$ column density. Therefore, in the models presented below, we first consider $\mathrm{C}_{2} \mathrm{H}_{2}$ alone and examine the effects of $\mathrm{HCN}$ later.

\subsection{Result of modeling with $\mathrm{C}_{2} \mathrm{H}_{2}$ layers and a dust shell}

We start from models consisting of one $\mathrm{C}_{2} \mathrm{H}_{2}$ layer with a constant temperature and density and an optically thin dust shell of amorphous carbon and $\mathrm{SiC}$, similar to the model used by Ohnaka et al. (2005). In this model, the temperature, outer radius, and the $\mathrm{C}_{2} \mathrm{H}_{2}$ column density of the layer are varied as free parameters. In addition, the inner radius of this layer is either set to be equal to $R_{\star}$ or varied as a free parameter to model a detached $\mathrm{C}_{2} \mathrm{H}_{2}$ layer. The central star itself is approximated with a blackbody of the effective temperature $T_{\text {eff }}$, which is fixed to $2800 \mathrm{~K}$ as in the dust shell models discussed above. Using the $\mathrm{C}_{2} \mathrm{H}_{2}$ opacity given above, the monochromatic intensity distribution of the $\mathrm{C}_{2} \mathrm{H}_{2}$ layer and the star is calculated as described in Ohnaka et al. (2004b). The intensity profile of the dust shell (excluding the central star) at each wavelength in the $N$ band is calculated with the mcsim_mpi code and ray-tracing method as in the above dust shell models, and this intensity profile is added to that resulting from the central star and the $\mathrm{C}_{2} \mathrm{H}_{2}$ layer. The inner boundary radius of the dust shell is set to $2.5 R_{\star}$, because the temperature at this radius (1500-1600 K) approximately corresponds to the condensation temperature of amorphous carbon grains. The spectrally convolved $N$-band visibilities are then computed as outlined in Sect. 3. However, it has turned out that these models cannot reproduce the shape of the $8 \mu \mathrm{m}$ absorption feature and the observed $N$-band visibilities simultaneously for the following reason. In these one-layer models, the $\mathrm{C}_{2} \mathrm{H}_{2}$ layer cannot be too extended, because otherwise the $v_{4}+v_{5}$ and $v_{5}$ bands would appear in emission, in disagreement with the observations. However, with a modest geometrical extension of the $\mathrm{C}_{2} \mathrm{H}_{2}$ layer, the angular sizes observed between 8 and $9 \mu \mathrm{m}$, which are remarkably larger than the photospheric size, cannot be reproduced.

Therefore, we examine models with a dust shell and two $\mathrm{C}_{2} \mathrm{H}_{2}$ layers, which are similar to the warm water vapor envelope model for oxygen-rich Miras considered by Ohnaka (2004a). In these models, the star is surrounded by two layers containing $\mathrm{C}_{2} \mathrm{H}_{2}$ with different temperatures $T_{\text {hot }}$ and $T_{\text {cool }}$, and the hot and cool layers extend to $R_{\text {hot }}$ and $R_{\text {cool }}$, respectively (see Fig. 10 for illustration of the model). The temperature and the density are assumed to be constant in each of these layers. The $\mathrm{C}_{2} \mathrm{H}_{2}$ column densities in the hot and cool layers are denoted as $N_{\text {hot }}$ and $N_{\text {cool }}$, respectively. The intensity profiles from two such layers are computed by summing up the contribution from each layer, and the total intensity profile is the sum of this component and the dust shell component with its inner boundary fixed to $2.5 R_{\star}$. From these intensity profiles, spectrally convolved visibilities are calculated as in the case of the above one-layer model. We search for a best-fit model within the following parameter range: $T_{\text {hot }}(\mathrm{K})=1300 \ldots 1800$ with $\Delta T_{\text {hot }}(\mathrm{K})=100, T_{\text {cool }}(\mathrm{K})=800$ $\ldots 1200 \mathrm{~K}$ with $\Delta T_{\text {cool }}(\mathrm{K})=100, R_{\text {hot }}\left(R_{\star}\right)=1.1 \ldots 1.6$ with $\Delta R_{\text {hot }}\left(R_{\star}\right)=0.1, R_{\text {cool }}\left(R_{\star}\right)=1.2 \ldots 2.0$ with $\Delta R_{\text {cool }}\left(R_{\star}\right)=0.1$ (with conditions $\left.R_{\text {hot }}<R_{\text {cool }}\right), \log N_{\text {hot }}\left(\mathrm{cm}^{-2}\right)=19 \ldots 22$, and $\log N_{\text {cool }}\left(\mathrm{cm}^{-2}\right)=18 \ldots 21$ with $\Delta \log N_{\text {hot }}=\Delta \log N_{\text {cool }}=$ 0.5 . The optical depth of the dust shell is varied between $\tau_{V}^{\text {amc }}$ $=0.1$ and 0.8 with $\Delta \tau_{V}^{\mathrm{amc}}=0.1$, while the fraction of $\mathrm{SiC}$, $f_{\mathrm{SiC}}=\tau_{V}^{\mathrm{SiC}} /\left(\tau_{V}^{\mathrm{amc}}+\tau_{V}^{\mathrm{SiC}}\right)$, is varied from 0.1 to 0.4 .

Not only the wavelength dependence of the observed visibilities but also the visibility shape as a function of spatial frequency constrained by the measurements at two different baseline lengths are crucial for this modeling. That is, the effect of the extended dust shell, which is over-resolved at the baseline lengths used in our MIDI observations, is to lower the total visibility by an amount equal to its fractional flux contribution at a given wavelength, as explained in Sect. 3. Therefore, the parameters of the dust shell roughly defines absolute visibility levels. On the other hand, the presence of the $\mathrm{C}_{2} \mathrm{H}_{2}$ layers makes the visibility (as a function of spatial frequency) steeper at higher spatial frequencies than models consisting of a bare central star and a dust shell. The $\mathrm{C}_{2} \mathrm{H}_{2}$ layers also give rise to absorption or emission at wavelengths between 8 and $9 \mu \mathrm{m}$ and longward of $\sim 11 \mu \mathrm{m}$, although in this latter wavelength range, the $\mathrm{C}_{2} \mathrm{H}_{2}$ feature is blended with the $\mathrm{SiC}$ emission feature. In this manner, it is possible to constrain the parameters of the hot and cool $\mathrm{C}_{2} \mathrm{H}_{2}$ layers as well as the dust shell.

Figures 6-8 show the best-fit models consisting of $\mathrm{C}_{2} \mathrm{H}_{2}$ layers and a dust shell for three epochs observed with MIDI, and the parameters of these best-fit models are given in Table 3. The figures demonstrate that the models can reproduce the observed $N$ band spectra, particularly the absorption feature between 8 and $9 \mu \mathrm{m}$. At the same time, the observed $N$-band visibilities - not 

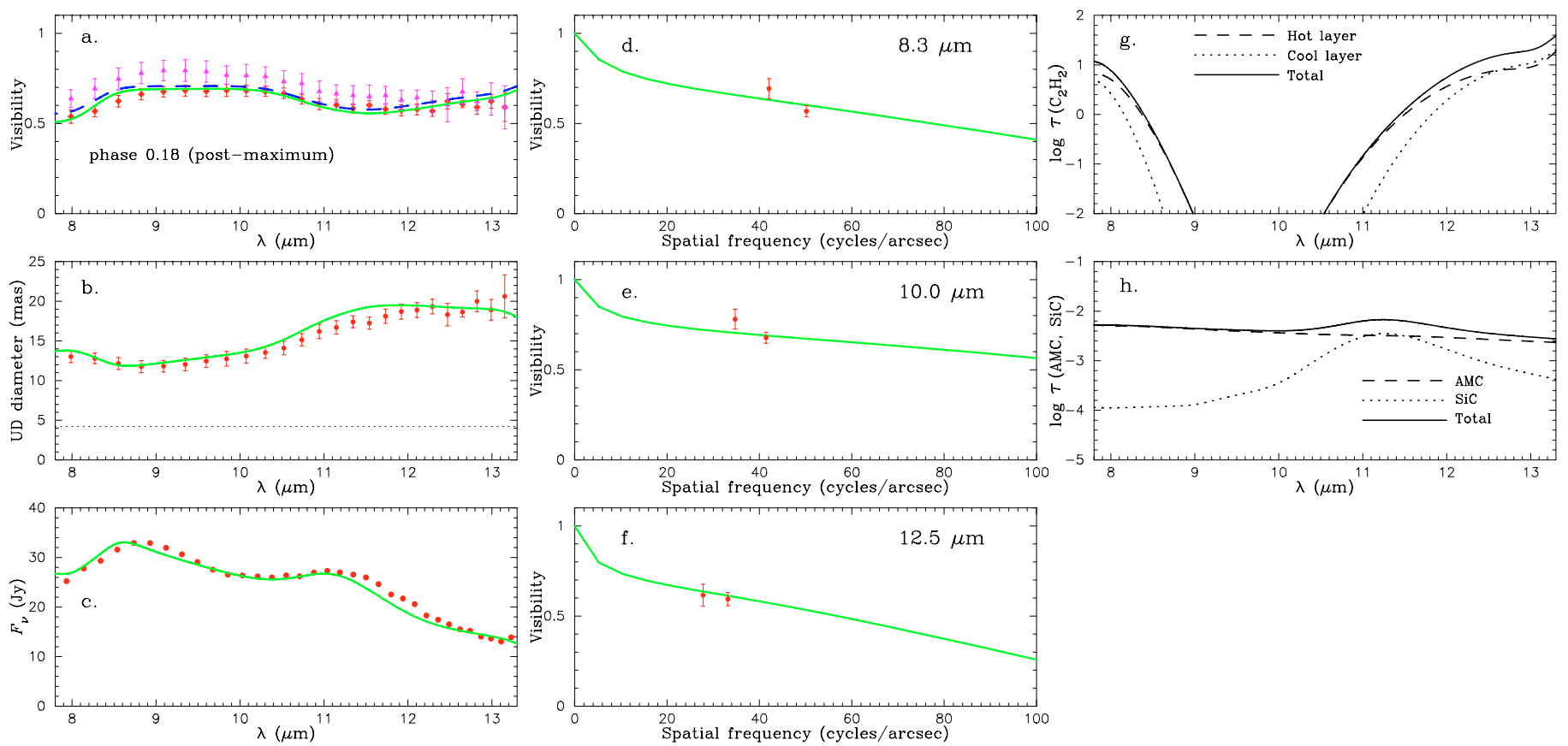

Fig. 6. Comparison between observational data at phase 0.18 (post-maximum) and the best-fit model consisting of $\mathrm{C}_{2} \mathrm{H}_{2}$ layers and a dust shell. The model parameters are given in Table 3. a) The filled diamonds and triangles represent the $N$-band visibilities measured at phase 0.18 with projected baseline lengths of 85.6 and $71.8 \mathrm{~m}$, respectively, while the solid and dashed lines represent the corresponding model visibilities. b) The filled circles represent the uniform-disk diameters observed at phase 0.18 , while the solid line represents the model prediction, which is derived by fitting the model visibilities predicted for baselines of 85.6 and $71.8 \mathrm{~m}$ with a uniform disk at each wavelength. The dotted line represents the photospheric angular diameter derived by fitting the model $N$-band spectrum to the observed data. c) Comparison of the observed and model $N$-band spectra. The filled circles represent the MIDI spectrum obtained at phase 0.18 , while the solid line represents the model prediction. d) $-\mathbf{f}$ ) The solid lines represent the model visibilities at 8.3, 10.0, and $12.5 \mu \mathrm{m}$ plotted as a function of spatial frequency. The observed data are plotted with the filled circles. g) The $\mathrm{C}_{2} \mathrm{H}_{2}$ optical depths of the hot and cool layers (convolved to a spectral resolution of 30). h) The optical depths for amorphous carbon (AMC) and $\mathrm{SiC}$ dust (convolved to a spectral resolution of 30).

only the absolute visibility levels but also their wavelength dependence - are also fairly reproduced by these models. The visibility increase from 8 to $10 \mu \mathrm{m}$, which is seen in all the data sets except for \#4 taken with the shortest baseline at minimum light, can be well explained by the models. The visibilities predicted at $12-13 \mu \mathrm{m}$, where the tail of the $\mathrm{C}_{2} \mathrm{H}_{2} \nu_{5}$ band is located, are also in reasonable agreement with the MIDI observations. These results suggest that the optically thick emission $\left(\tau_{\text {line }} \approx 1-100\right.$, see Figs. $6 \mathrm{~g}, 7 \mathrm{~g}$, and $8 \mathrm{~g}$ ) from the $\mathrm{C}_{2} \mathrm{H}_{2}$ layers, together with the dust emission, is responsible for the $N$-band angular size significantly larger than the photospheric size and that $\mathrm{C}_{2} \mathrm{H}_{2}$ plays a role similar to $\mathrm{H}_{2} \mathrm{O}$ in oxygen-rich environments. Also, as will be discussed below, this optically thick emission plays an important role in the interpretation of the controversial apparent depth of the 7 and $14 \mu \mathrm{m} \mathrm{C}_{2} \mathrm{H}_{2}$ features outlined in Sect. 1. We note that as in the case of the dust shell models discussed above, the model visibilities are scaled using the angular radius of the central star (stellar continuum angular radius) derived by fitting the model $N$-band spectra to those observed. The stellar photospheric angular sizes derived in this manner are also given in Table 3. In this method, the uncertainty of the stellar diameter is primarily determined by the uncertainties of the absolutely calibrated $N$-band spectra of $\sim 10-20 \%$. Based on this, we estimate the error of the stellar diameter to be \pm 0.3 mas. It is noteworthy that while the observed uniform-disk diameters are the smallest at minimum light (see Fig. 2d), the stellar continuum diameter does not show such a phase dependence (almost constant within the uncertainty or may marginally be the largest at minimum light). This means that the $N$-band apparent size is largely governed by the opacity and the geometrical extension of the $\mathrm{C}_{2} \mathrm{H}_{2}$ layers and the dust shell, and does not reflect the size variation of the underlying photosphere.

The effects of the uncertainty of the effective temperature of the central star on the derived parameters are checked by calculating models with $T_{\text {eff }}=3000$ and $2600 \mathrm{~K}$ and included in the uncertainty of the derived parameters. However, these effects have turned out to be relatively minor, because the $\mathrm{C}_{2} \mathrm{H}_{2}$ layers, particularly the hot layer, are mostly optically thick, masking the underlying central star. We also examine the effects of HCN on model visibilities and spectra by adding the HCN contribution to the best-fit models, using the line list of Harris et al. (2002) and the Gaussian line profile with a $F W H M$ of $5 \mathrm{~km} \mathrm{~s}^{-1}$. However, since the spectral feature of $\mathrm{HCN}$ is blended with the $\mathrm{C}_{2} \mathrm{H}_{2} v_{5}$ band and the $\mathrm{SiC}$ dust feature, we cannot put a strong constraint on the HCN column densities, and only the upper limits of the HCN column densities are derived as given in Table 3.

Figure 9 shows the derived properties of the $\mathrm{C}_{2} \mathrm{H}_{2}$ layers and the dust shell as functions of phase, and a simple cartoon of the models at the three phases is shown in Fig. 10 to illustrate the size of the $\mathrm{C}_{2} \mathrm{H}_{2}$ layers and the dust shell and their phase dependence. The radius of the $\operatorname{cool~} \mathrm{C}_{2} \mathrm{H}_{2}$ layer shows noticeable variations: the layer is more extended at post-maximum (phase 0.18 ) and post-minimum (phase 0.65 ) than at minimum light (phase 0.49). Furthermore, the column density of the cool $\mathrm{C}_{2} \mathrm{H}_{2}$ layer appears to be the smallest at minimum light, although the uncertainties of the derived column densities are rather large. The temperature of the cool layer is found to exhibit no temporal variation within the uncertainties of our modeling. However, it is not straightforward to interpret these temporal variations of the radii as the oscillating motion of the $\mathrm{C}_{2} \mathrm{H}_{2}$ layer. That is, the 

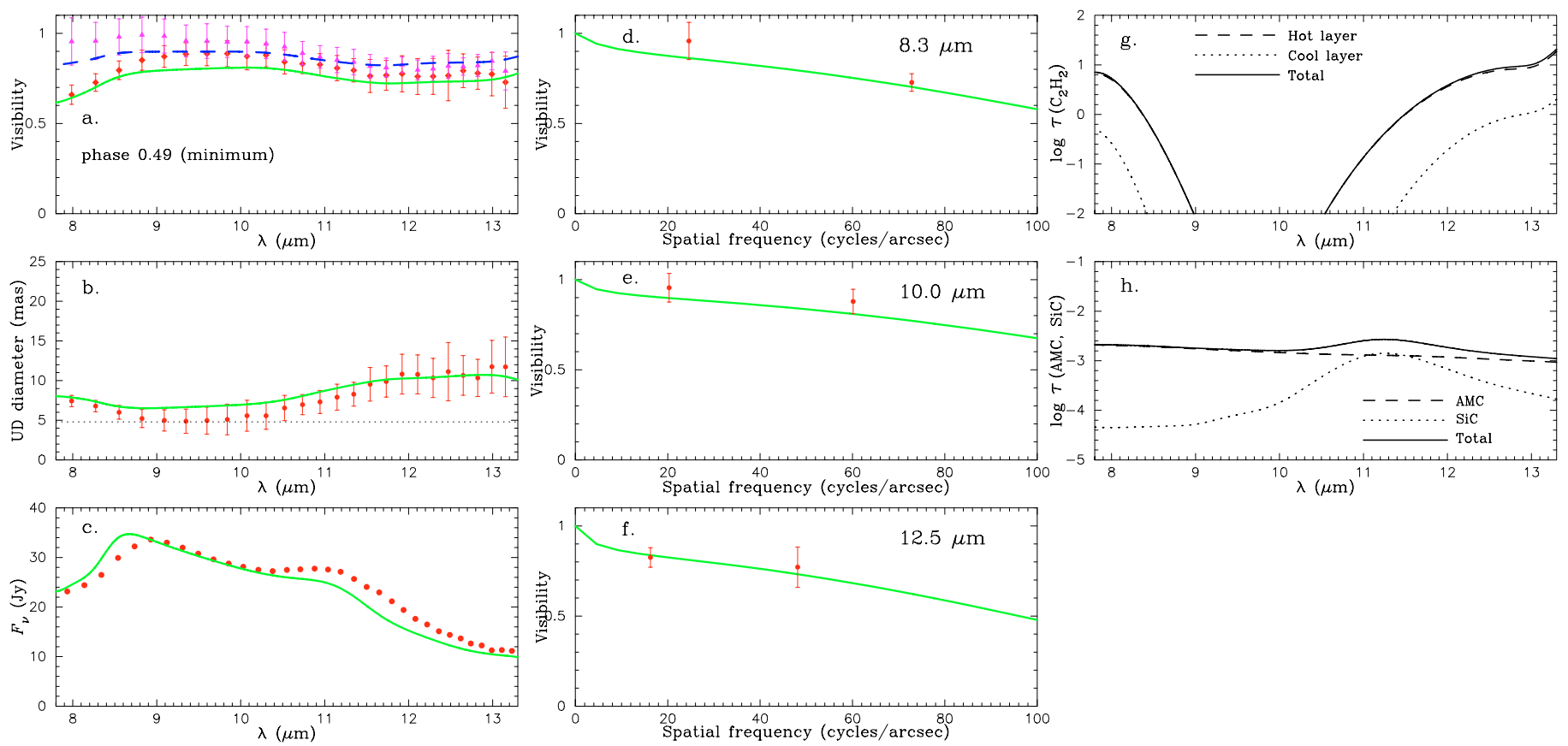

Fig. 7. Same as Fig. 6, but for phase 0.49 (minimum light). In the panel a), the filled diamonds and triangles represent the $N$-band visibilities measured with projected baseline lengths of 124.2 and $41.9 \mathrm{~m}$, respectively, while the solid and dashed lines represent the corresponding model visibilities. In the panel b), the solid line represents the model prediction, which is derived by fitting the model visibilities predicted for baselines of 124.2 and $41.9 \mathrm{~m}$ with uniform disks. In the panel $\mathbf{g}$ ), the solid and dashed lines almost entirely overlap.
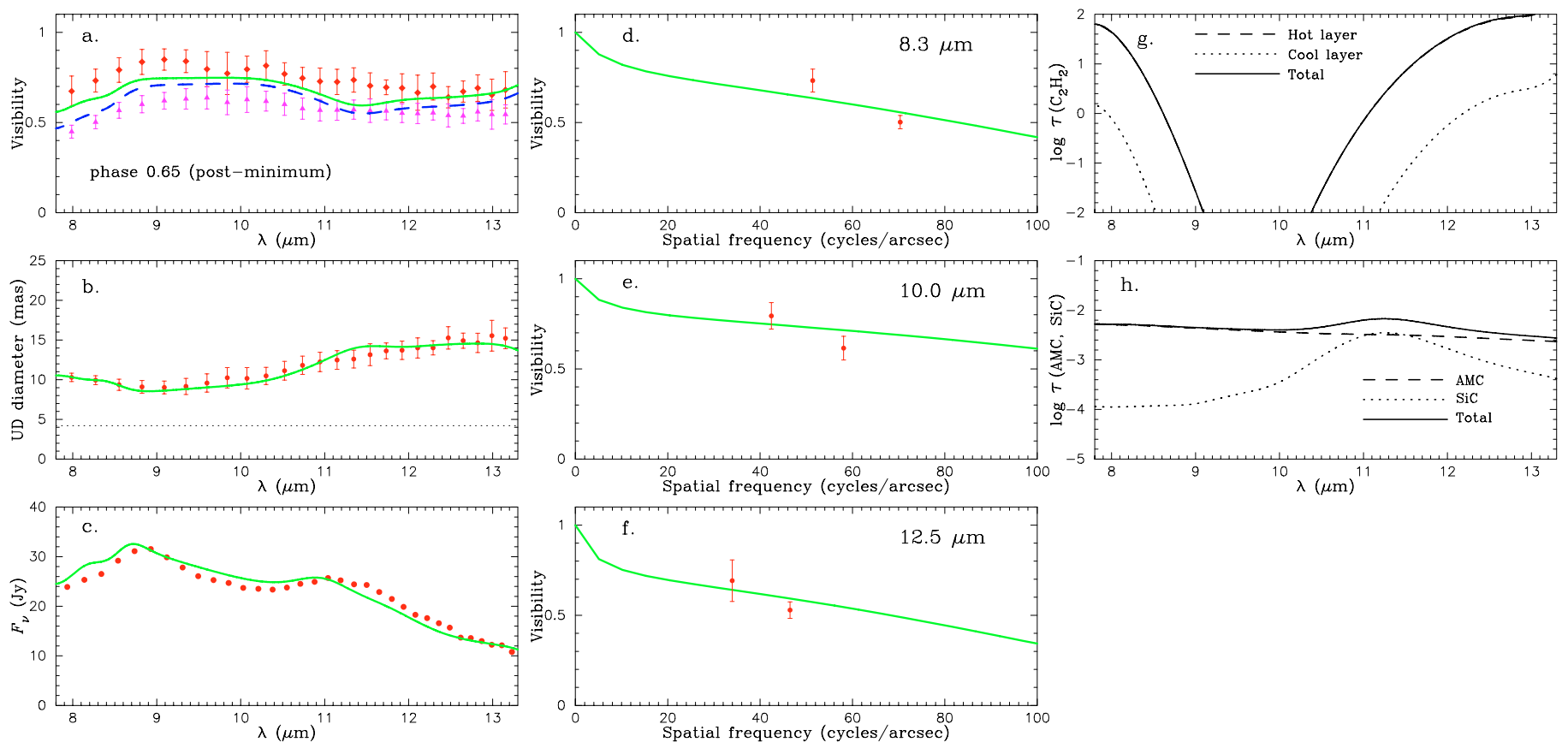

Fig. 8. Same as Fig. 6, but for phase 0.65 (post-minimum). In the panel a), the filled diamonds and triangles represent the $N$-band visibilities measured with projected baseline lengths of 87.6 and $120.0 \mathrm{~m}$, respectively, while the solid and dashed lines represent the corresponding model visibilities. In the panel b), the solid line represents the model prediction, which is derived by fitting the model visibilities predicted for baselines of 87.6 and $120.0 \mathrm{~m}$ with uniform disks. In the panel $\mathbf{g}$ ), the solid and dashed lines almost entirely overlap.

smaller radius of the cool layer at minimum light (phase 0.49 ) does not necessarily mean that $\mathrm{C}_{2} \mathrm{H}_{2}$ gas shrinks from phase 0.18 to 0.49 and bounces back from phase 0.49 to 0.65 . As discussed in Sect. 5 , it is also possible that $\mathrm{C}_{2} \mathrm{H}_{2}$ layers may be associated with the successive passage of shock waves propagating outward and that the observed temporal variations of the radii and column densities of the $\mathrm{C}_{2} \mathrm{H}_{2}$ layers are simply a series of "snapshots" of such a dynamical atmosphere.

The variation of the $\mathrm{C}_{2} \mathrm{H}_{2}$ column densities may appear to contradict the phase dependence of the strength of the $8-9 \mu \mathrm{m}$ feature mentioned in Sect. 2: the absorption becomes stronger at minimum light (phase 0.49 ) than at post-maximum (phase 0.18). 

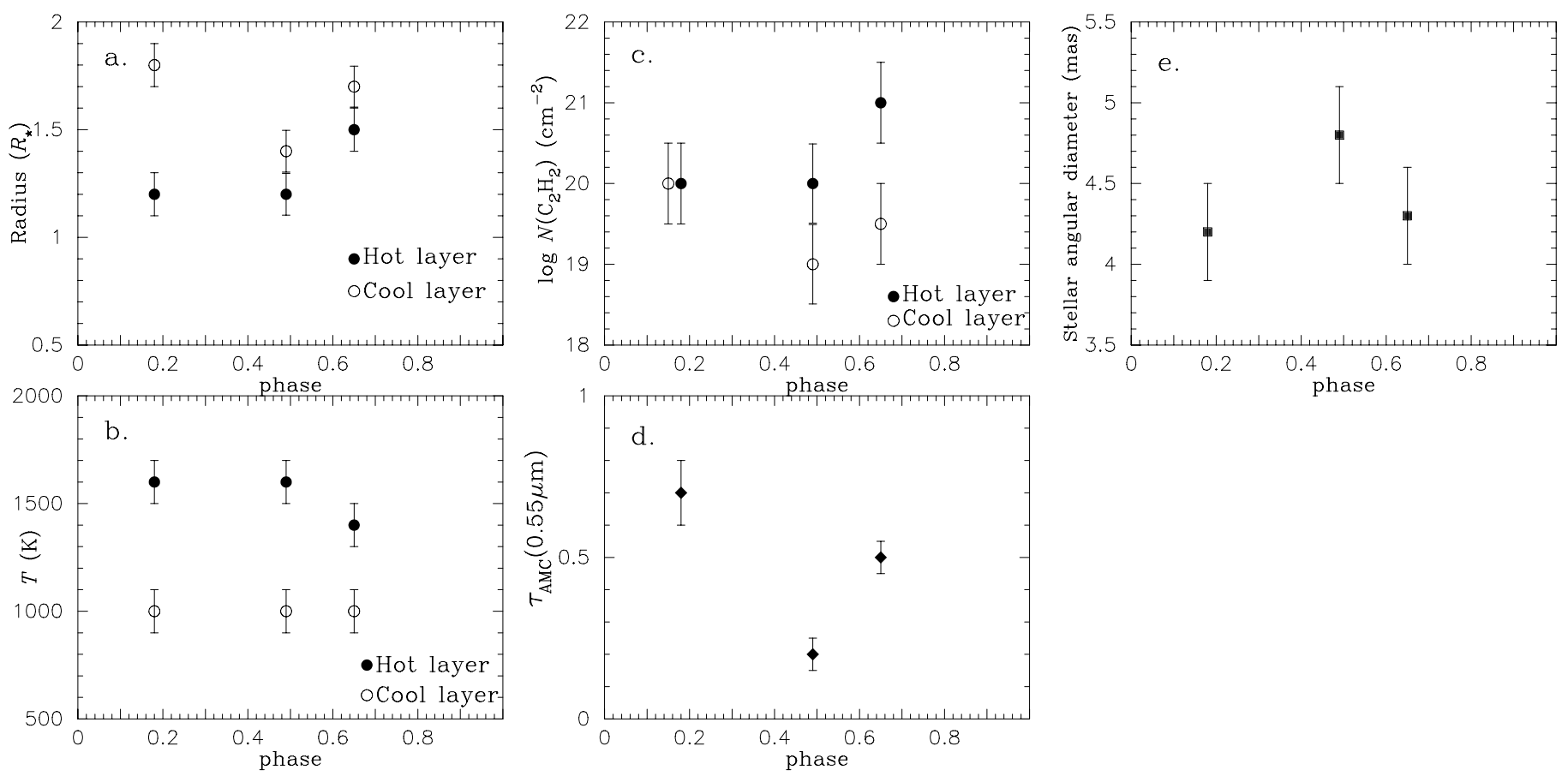

Fig. 9. Temporal variations of the radii, temperatures, and column densities of the hot and $\operatorname{cool} \mathrm{C}_{2} \mathrm{H}_{2}$ layers a), b), and c), the optical depth of amorphous carbon dust in the dust shell $\mathbf{d}$ ), and the continuum stellar angular diameter $\mathbf{e}$ ).

Table 3. Derived parameters of the $\mathrm{C}_{2} \mathrm{H}_{2}(+\mathrm{HCN})$ layers and the dust shell around V Oph. The stellar angular diameter $\left(2 R_{\star} / d\right)$, where $d$ is the distance of $\mathrm{V}$ Oph, was derived by fitting the model $N$-band spectra to those observed.

\begin{tabular}{lccc}
\hline \hline & Phase 0.18 & Phase 0.49 & Phase 0.65 \\
\hline$T_{\text {hot }}(\mathrm{K})$ & $1600 \pm 100$ & $1600 \pm 100$ & $1400 \pm 100$ \\
$R_{\text {hot }}\left(R_{\star}\right)$ & $1.2 \pm 0.1$ & $1.2 \pm 0.1$ & $1.5 \pm 0.1$ \\
$\log N_{\text {hot }}\left(\mathrm{C}_{2} \mathrm{H}_{2}\right)\left(\mathrm{cm}^{-2}\right)$ & $20.0 \pm 0.5$ & $20 \pm 0.5$ & $21 \pm 0.5$ \\
$T_{\text {cool }}(\mathrm{K})$ & $1000 \pm 100$ & $1000 \pm 100$ & $1000 \pm 100$ \\
$R_{\text {cool }}\left(R_{\star}\right)$ & $1.8 \pm 0.1$ & $1.4 \pm 0.1$ & $1.7 \pm 0.1$ \\
$\log N_{\text {cool }}\left(\mathrm{C}_{2} \mathrm{H}_{2}\right)\left(\mathrm{cm}^{-2}\right)$ & $20.0 \pm 0.5$ & $19 \pm 0.5$ & $19.5 \pm 0.5$ \\
$\tau_{V}^{\text {amc }}$ & $0.7 \pm 0.1$ & $0.2 \pm 0.05$ & $0.5 \pm 0.05$ \\
$f_{\mathrm{SiC}}=\tau_{V}^{\mathrm{SiC}} /\left(\tau_{V}^{\mathrm{amc}}+\tau_{V}^{\mathrm{SiC}}\right)$ & $0.2 \pm 0.1$ & $0.2 \pm 0.1$ & $0.2 \pm 0.1$ \\
$\log N_{\text {hot }}(\mathrm{HCN})\left(\mathrm{cm}^{-2}\right)$ & $\leq 20$ & $\leq 19$ & $\leq 21$ \\
$\log N_{\text {cool }}(\mathrm{HCN})\left(\mathrm{cm}^{-2}\right)$ & $\leq 20$ & $\leq 18$ & $\leq 21$ \\
Stellar angular & $4.2 \pm 0.3$ & $4.8 \pm 0.3$ & $4.3 \pm 0.3$ \\
$\operatorname{diameter}(\mathrm{mas})$ & & & \\
\hline
\end{tabular}

This seemingly inconsistent result can be explained as follows. At maximum light, the greater geometrical extension of the $\mathrm{C}_{2} \mathrm{H}_{2}$ layers results in the higher contribution of the emission from the extended part of the layers (i.e., beyond the central star's limb). In other words, the absorption is partially, though not entirely, filled in by this emission component. On the other hand, at minimum light, the smaller geometrical extension of the $\mathrm{C}_{2} \mathrm{H}_{2}$ layers makes the contribution of this extended emission component smaller, which results in stronger absorption.

The hot $\mathrm{C}_{2} \mathrm{H}_{2}$ layer shows a temporal variation somewhat different from that of the cool layer. The hot layer becomes more extended, denser, and a little cooler at phase 0.65 compared to the preceding two phases. The dust shell also shows noticeable temporal variations: its optical depth is the smallest at minimum light, while the fraction of $\mathrm{SiC}$ dust does not show significant changes within the uncertainty of our modeling. This phase dependence of the optical depth of the dust shell is similar to that found in optically bright oxygen-rich Miras $o$ Cet and Z Cyg by Suh (2004), who concludes that stronger stellar winds near maximum light (higher stellar luminosities) lead to greater dust production. We will discuss this point further in Sect. 5.

We note that while the inner boundary radius of the dust shell in the above modeling is fixed by the condensation temperature of amorphous carbon, the stellar pulsation may lead to departure from radiative equilibrium, and dust may form closer to the star than it would in radiative equilibrium (Willson 2000). In order to assess this possibility, we compute dust shell models with an inner boundary radius equal to $R_{\text {cool }}$ (i.e., no gap between the cool $\mathrm{C}_{2} \mathrm{H}_{2}$ layer and the dust shell inner boundary) by artificially imposing an upper limit of $1600 \mathrm{~K}$ on the dust temperatures. We find out that these ad hoc "non-equilibrium" models can reproduce the observed $N$-band visibilities and spectra at three phases if the optical depths are increased, with the parameters of the $\mathrm{C}_{2} \mathrm{H}_{2}$ layers kept the same as the above models. For example, the $\mathrm{C}_{2} \mathrm{H}_{2}+$ dust shell model with $\tau_{V}^{\mathrm{amc}}=1.0$ and $\tau_{V}^{\mathrm{SiC}}=0.25 \mathrm{can}$ provide a fit as good as the model shown in Fig. 6. This means that the inner boundary radius of the dust shell cannot be well constrained, because the visibility component of the dust shell, which appears as a steep drop at the lowest spatial frequencies, is not sampled by our MIDI observations using rather long baseline lengths. It would also be possible that dust coexists with the $\mathrm{C}_{2} \mathrm{H}_{2}$ gas. In this case, the innermost part of the dust shell would be at least partially masked by the optically thick $\mathrm{C}_{2} \mathrm{H}_{2}$ gas, which would mimic the dust shell with its inner boundary equal to $R_{\text {cool }}$. In addition, the non-LTE grain formation calculation of Kozasa et al. (1996) shows that SiC grains form first and then amorphous carbon mantles form around the $\mathrm{SiC}$ cores at low mass loss rates, which is not considered in our present model. These dust shell properties could be better constrained by observations with much shorter baselines ( $\$ 20 \mathrm{~m})$.

There are still discrepancies between the best-fit models and the observed data. At phase 0.18 and 0.65 , the predicted visibilities (as a function spatial frequency) are still somewhat too 

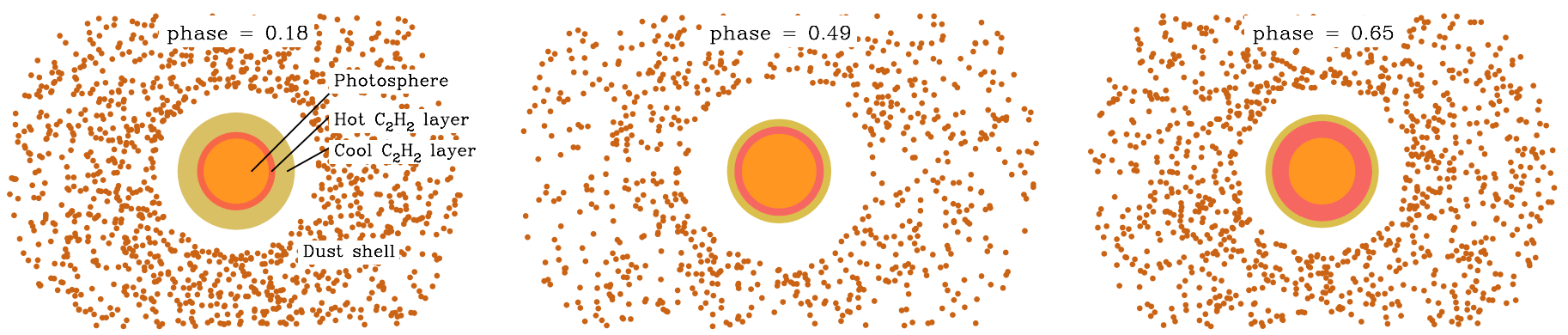

Fig. 10. A simple cartoon illustrating the best-fit models at phases $0.18,0.49$, and 0.65 . The sizes of the components at three phases are to scale except for the outer radius of the dust shell.

flat compared to the MIDI visibilities measured at two baseline lengths. This means that the $\mathrm{C}_{2} \mathrm{H}_{2}$ layers may be larger than in these models. In the framework of our two-layer modeling, however, adoption of larger radii for the hot and/or cool $\mathrm{C}_{2} \mathrm{H}_{2}$ layers turns the absorption feature between 8 and $9 \mu \mathrm{m}$ to emission. Moreover, while we assume for simplicity that the hot $\mathrm{C}_{2} \mathrm{H}_{2}$ layer extends down to the photosphere and the hot and $\operatorname{cool~} \mathrm{C}_{2} \mathrm{H}_{2}$ layers are in contact, it is possible that these $\mathrm{C}_{2} \mathrm{H}_{2}$ layers are detached and geometrically thin. This may also explain the discrepancy between the models and the observed data. In any case, our two-layer model with constant temperatures and densities is a rather simplistic representation of real density and temperature distributions, and more sophisticated models of the $\mathrm{C}_{2} \mathrm{H}_{2}$ layers constrained by further complimentary observational data may reproduce the MIDI data better. For example, mid-infrared high-resolution spectra of the $\mathrm{C}_{2} \mathrm{H}_{2} v_{4}+v_{5}$ and $v_{5}$ bands would shed more light on the physical properties of the $\mathrm{C}_{2} \mathrm{H}_{2}$ layers, as $11 \mu \mathrm{m}$ high-resolution spectra have proven to be strong observational constraints in the case of oxygen-rich Mira variables (e.g., Ohnaka 2004a; Ohnaka 2006b). Also, although we assume LTE in the calculation of the molecular opacities, it is necessary to examine non-LTE effects when an extensive high-temperature line list of $\mathrm{C}_{2} \mathrm{H}_{2}$ becomes available.

\section{Discussion}

Yamamura et al. (1997) analyzed the mid-infrared spectra of several carbon stars obtained with ISO/SWS and derived the $\mathrm{C}_{2} \mathrm{H}_{2}$ column densities and temperatures from the $13.7 \mu \mathrm{m}$ feature. The $\mathrm{C}_{2} \mathrm{H}_{2}$ column densities of $10^{18 \pm 0.5} \mathrm{~cm}^{-2}$ are somewhat lower than those we derived for the cool $\mathrm{C}_{2} \mathrm{H}_{2}$ layer, while the temperatures of $1200 \pm 300 \mathrm{~K}$ that they derived are in fair agreement with those of the cool layer of our models. They measured the strength of the $13.7 \mu \mathrm{m}$ feature with respect to the local continuum levels, which are affected by the broad (hot) $\mathrm{C}_{2} \mathrm{H}_{2}$ absorption component as well as dust emission. Therefore, the $\mathrm{C}_{2} \mathrm{H}_{2}$ column density and temperature derived by Yamamura et al. (1997) may refer to the cooler, outermost layers.

Nowotny et al. (2005a,b) have presented a self-consistent dynamical model including dust formation processes for the carbon-rich Mira S Cep $\left(\dot{M} \approx 10^{-6} M_{\odot} \mathrm{yr}^{-1}\right)$. They demonstrate that their model can qualitatively reproduce the observed temporal variations of the radial velocity and line profiles of infrared molecular lines such as $\mathrm{CO}$ and $\mathrm{CN}$. The density distribution of their model shows a number of step-like features resulting from the formation of shock fronts propagating outward. It is plausible that polyatomic molecules such as $\mathrm{C}_{2} \mathrm{H}_{2}$ and $\mathrm{HCN}$ form behind shock fronts - possibly at the innermost ones where density is high - just as $\mathrm{H}_{2} \mathrm{O}$ shells form in oxygen-rich Miras (Tej et al. 2003). Therefore, it is possible that the observed temporal variation of the radii of the $\mathrm{C}_{2} \mathrm{H}_{2}$ layers results from changes of the positions of $\mathrm{C}_{2} \mathrm{H}_{2}$ formation behind shock fronts. This means that the cool layer extending to $\sim 1.8 R_{\star}$ at phase 0.18 moves farther outward and may no longer contribute to the $N$-band flux at phase 0.49 . And the small radius of the cool layer obtained at phase 0.49 suggests that a new $\mathrm{C}_{2} \mathrm{H}_{2}$ formation front may just be emerging at a smaller radius and that this front may build up and move outward from phase 0.49 to 0.65 .

The phase dependence of the grain optical depths we derived for V Oph can also be interpreted in a similar manner. The model presented by Nowotny et al. (2005a,b) shows that dust is produced in discrete shells behind shock fronts where the density is sufficiently high and the temperature is below condensation temperature. If such a hot dust layer is responsible for the higher grain optical depths we derived for phase 0.18 (post-maximum light), the dust layer is expected to expand from phase 0.18 to 0.49 (minimum light) and become diluted. At minimum light, a new (inner) dust layer can be forming, but still with a low degree of condensation, which can qualitatively explain the smallest grain optical depths at minimum light. When this new dust layer has fully grown, it can provide higher grain optical depths at post-minimum phases. However, as explained above, our present MIDI data cannot constrain the inner boundary radius of the dust shell strongly enough to detect the apparent motion of dust layers expected from this scenario. We also note that the mass loss rate of $\mathrm{V} \mathrm{Oph}$ is of the order of $10^{-8} M_{\odot} \mathrm{yr}^{-1}$, which is by two orders of magnitude lower than that of S Cep. It is necessary to compare with dynamical models computed with parameters corresponding to $\mathrm{V}$ Oph for a better understanding of the physical processes responsible for molecule and dust formation close to the star: whether the extended $\mathrm{C}_{2} \mathrm{H}_{2}$ layers can be explained by dust-driven winds triggered by large-amplitude stellar pulsation alone or some other mechanism is operating such as Alfvén wave-driven winds (e.g., Vidotto \& Janteco-Pereira 2006; Suzuki 2006).

The result that our two-layer models can reproduce the observed $\mathrm{C}_{2} \mathrm{H}_{2}$ absorption feature between 8 and $9 \mu \mathrm{m}$ implies that the emission from these dense, geometrically extended $\mathrm{C}_{2} \mathrm{H}_{2}$ layers may be responsible for the observed weakness of the $\mathrm{C}_{2} \mathrm{H}_{2}$ and $\mathrm{HCN}$ absorption features at 7 and $14 \mu \mathrm{m}$. While the dust thermal emission also contributes to rendering these molecular features weak, the low mass loss rate of $\mathrm{V}$ Oph suggests that the optically thick emission from the $\mathrm{C}_{2} \mathrm{H}_{2}$ layers plays a major role at least in optically bright Mira-type carbon stars. In order to confirm this, it would be crucial to compare model predictions with observed strengths of the $\mathrm{C}_{2} \mathrm{H}_{2}$ and $\mathrm{HCN}$ features and their temporal variations at $3 \mu \mathrm{m}$ (accessible from the ground) as well as 7 and $14 \mu \mathrm{m}$ (with Spitzer and/or AKARI). 


\section{Conclusions}

We have carried out multi-epoch $N$-band spectro-interferometric observations of the carbon-rich Mira V Oph and spatially resolved the object between 8 and $13 \mu \mathrm{m}$. The observed $N$-band uniform-disk diameters are by a factor of 2-3 larger than the estimated stellar continuum size, as has already been established for oxygen-rich Miras. The visibilities obtained at phases 0.18 , 0.49 , and 0.65 reveal that the object appears smaller at phase 0.49 (minimum light) than at phases 0.18 and 0.65 . Our simple modeling shows that the observed $\mathrm{N}$-band visibilities and spectra can be reproduced by optically thick $\mathrm{C}_{2} \mathrm{H}_{2}$ layers and a dust shell consisting of amorphous carbon and $\mathrm{SiC}$. The $\operatorname{cool~} \mathrm{C}_{2} \mathrm{H}_{2}$ layer is more extended and denser at phases 0.18 and 0.65 than at 0.49 , while the hot $\mathrm{C}_{2} \mathrm{H}_{2}$ layer becomes more extended and denser at phase 0.65 with no noticeable change between phase 0.18 and 0.49. This modeling also implies that the weakness of the $\mathrm{C}_{2} \mathrm{H}_{2}$ and $\mathrm{HCN}$ absorption features at 7 and $14 \mu \mathrm{m}$ observed in carbon-rich Miras can be explained by emission from these extended, dense $\mathrm{C}_{2} \mathrm{H}_{2}$ (and $\mathrm{HCN}$ ) layers, together with dust emission. Since our modeling is based on the $\mathrm{C}_{2} \mathrm{H}_{2}$ opacity computed with the RRHO band-model approximation, it is essential to examine the present results with an extensive high-temperature line list of $\mathrm{C}_{2} \mathrm{H}_{2}$ in the future. However, while the $\mathrm{C}_{2} \mathrm{H}_{2}$ column densities can be directly affected by the uncertainty of the $\mathrm{C}_{2} \mathrm{H}_{2}$ opacity, the trend of the phase-dependent variation of the $\mathrm{C}_{2} \mathrm{H}_{2}$ column densities found in the present work is less likely to be very affected.

In order to better understand the phase and cycle dependence of the physical properties of the outer atmosphere and the dust shell, it is indispensable to carry out near-infrared interferometric observations simultaneously with MIDI observations. Such coordinated observations are feasible with VLTI, using MIDI and the near-infrared beam combiner, AMBER, which is capable of measuring visibilities in the $J, H$, and $K$ bands with three different spectral resolutions of 75,1500 , and 12000 . With the highest spectral resolution, it is possible to measure the stellar continuum diameter, although not entirely free from molecular line contamination, and to obtain a more comprehensive picture of how the outer atmosphere and the dust shell respond to stellar pulsation.

Acknowledgements. We thank the ESO VLTI team on Paranal and in Garching and the MIDI team for carrying out the observations and making the data reduction software publicly available. We are also indebted to the referee, B. Mennesson, for his constructive comments. We acknowledge with thanks the variable star observations from the AAVSO International Database contributed by observers worldwide and used in this research.

\section{References}

Aoki, W., Tsuji, T., \& Ohnaka, K. 1998, A\&A, 340, 222 Aoki, W., Tsuji, T., \& Ohnaka, K. 1999, A\&A, 350, 945 Bergeat, J., Knapik, A., \& Rutily, B. 2001, A\&A, 369, 178
Chesneau, O., Meilland, A., Rivinius, T., et al. 2005a, A\&A, 435, 275

Chesneau, O., Verhoelst, T., Lopez, B., et al. 2005b, A\&A, 435, 563

Cohen, M., Walker, R. G., Carter, B., et al. 1999, AJ, 117, 1864

Fedele, D., Wittkowski, M., Paresce, F., et al. 2005, A\&A, 431, 1019

Gautchy-Loidl, R., Höfner, S., Jørgensen, U. G., \& Hron, J. 2004, A\&A, 422, 289

Groenewegen, M. A. T. 2006, A\&A, 448, 181

Groenewegen, M. A. T., Whitelock, P. A., Smith, C. H., \& Kerschbaum, F. 1998, MNRAS, 293, 18

Harris, G., Polyansky, O. L., \& Tennyson, J. 2002, ApJ, 578, 657

Hinkle, K. H., \& Barnes, T. G. 1979, ApJ, 227, 923

Hron, J., Loidl, R., Höfner, S., et al. 1998, A\&A, 335, L69

Ireland, M., Tuthill, P., Robertson, G., et al. 2004, in Variable Stars in the Local Group, ed. D. W. Kurtz \& K. Pollard, ASP. Conf. Ser., 317, 327

Jaffe, W. 2004, SPIE Proc., 5491, 715

Jørgensen, U. G., Hron, J., \& Loidl, R. 2000, A\&A, 356, 253

Kabbadj, Y., Herman, M., Di Lonardo, G., \& Fusina, L. 1991, J. Mol. Sp., 150, 535

Kervella, P., Mérand, A., Perrin, G., \& Coudé du Foresto, V. 2006, A\&A, 448, 623

Kozasa, T., Dorschner, J., Henning, Th., \& Stognienko, R. 1996, A\&A, 307, 551 Landolt, A. U. 1968, PASP, 80, 680

Landolt, A. U. 1973, PASP, 85, 625

Leinert, Ch., van Boekel, R., Waters, L. B. F. M., et al. 2004, A\&A, 423, 537

Mennesson, B., Perrin, G., Chagnon, G., et al. 2002, ApJ, 579, 446

Noguchi, K., Kawara, K., Kobayashi, Y., Okuda, H., \& Sato, S. 1981, PASJ, 33, 373

Nowotny, W., Aringer, B., Höfner S., Gautschy-Loidl, R., \& Windsteig, W. 2005a, A\&A, 437, 273

Nowotny, W., Lebzelter, T., Hron, J., \& Höfner, S. 2005b, A\&A, 437, 285

Ohnaka, K. 2004a, A\&A, 424, 1011

Ohnaka, K. 2004b, A\&A, 421, 1149

Ohnaka, K., \& Tsuji, T. 1996, A\&A, 310, 933

Ohnaka, K., Bergeat, J., Driebe, T., et al. 2005, A\&A, 429, 1057

Ohnaka, K., Driebe, T., Hofmann, K.-H., et al. 2006a, A\&A, 445, 1015

Ohnaka, K., Scholz, M., \& Wood, P. R. 2006b, A\&A, 446, 1119

Pégourié, B. 1988, A\&A, 194, 335

Perrin, G., Ridgway, S. T., Mennesson, B., et al. 2004, A\&A, 426, 279

Poncelet, A., Perrin, G., \& Sol, H. 2006, A\&A, 450, 483

Przygodda, F., Chesneau, O., Graser, U., Leinert, Ch., \& Morel, S. 2003, Ap\&SS, 286,85

Quanz, S. P., Henning, Th., Bouwman, J., Ratzka, Th., \& Leinert, Ch. 2006, ApJ, 648,472

Rouleau, F., \& Martin, P. G. 1991, ApJ, 377, 526

Samus, N. N., Durlevich, O. V, et al. 2004, Combined General Catalogue of Variable Stars

Savage, B. D., \& Mathis, J.S. 1979, ARA\&A, 17, 73

Suh, K.-W. 2004, ApJ, 615, 485

Suzuki, T. K. 2006, ApJ, submitted [arXiv: astro-ph/0608195]

Tej, A., Lançon, A., \& Scholz, M. 2003, A\&A, 401, 347

Thompson, R. R., Creech-Eakman, M. J., \& van Belle, G. T. 2002, ApJ, 577, 447

Tsuji, T. 1984, A\&A, 134, 24

Tsuji, T., Ohnaka K., Aoki, W., \& Yamamura, I. 1997, A\&A, 320, L1

van Belle, G. T., Dyck, H. M., Thompson, R. R., Benson, J. A., \& Kannappan, S. J. 1997, AJ, 114, 2150

Vidotto, A. A., \& Janteco-Pereira, V. 2006, ApJ, 639, 416

Walker, A. R. 1979, SAAO Circ. 1, 112

Whitelock, P., Marang, F., \& Feast, M. 2000, MNRAS, 319, 728

Willson, L. A. 2000, ARA\&A, 38, 573

Woodruff, C., Eberhardt, M., Driebe, T., et al. 2004, A\&A, 421, 703

Yamamura, I., de Jong, T., Justtanont, K., Cami, J., \& Waters, L. B. F. M. 1997, Ap\&SS, 255, 351

Yamamura, I., de Jong, T., \& Cami, J. 1999, A\&A, 348, L55 